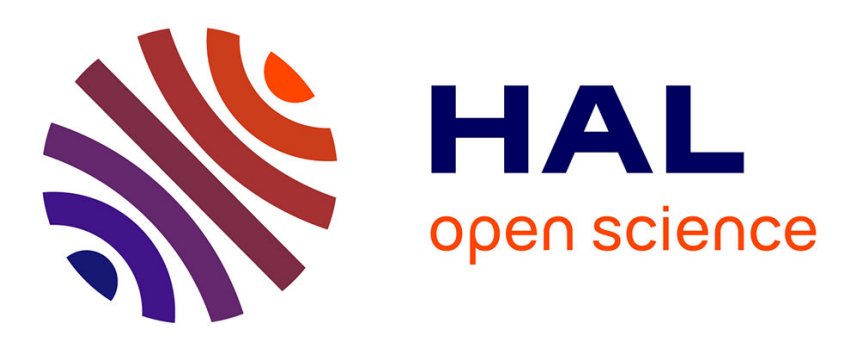

\title{
14-3-3 regulates life span by both DAF-16-dependent and -independent mechanisms in Caenorhabditis elegans.
}

\author{
Caroline Araiz, Marie-Thérèse Château, Simon Galas
}

\section{To cite this version:}

Caroline Araiz, Marie-Thérèse Château, Simon Galas. 14-3-3 regulates life span by both DAF-16dependent and -independent mechanisms in Caenorhabditis elegans.. Experimental Gerontology, 2008, 43 (6), pp.505-19. 10.1016/j.exger.2008.03.001 . hal-00345888v2

\section{HAL Id: hal-00345888 \\ https://hal.science/hal-00345888v2}

Submitted on 12 Dec 2008

HAL is a multi-disciplinary open access archive for the deposit and dissemination of scientific research documents, whether they are published or not. The documents may come from teaching and research institutions in France or abroad, or from public or private research centers.
L'archive ouverte pluridisciplinaire HAL, est destinée au dépôt et à la diffusion de documents scientifiques de niveau recherche, publiés ou non, émanant des établissements d'enseignement et de recherche français ou étrangers, des laboratoires publics ou privés. 


\title{
14-3-3 regulates life span by both DAF-16-dependent and independent mechanisms in Caenorhabditis elegans.
}

\author{
Caroline Araiz ${ }^{1}$, Marie-Thérèse Château ${ }^{1,2}$ and Simon Galas ${ }^{1,2}$ \\ ${ }^{1}$ CRBM- CNRS, 1919 route de Mende, 34293 Montpellier cedex 5, France; \\ ${ }^{2}$ Université Montpellier1, Faculté de Pharmacie, 15 Avenue Charles Flahault - B.P. 14491, \\ 34093 Montpellier cedex 5.
}

Keywords: [C. elegans, ageing, Insulin/IGF-1/DAF-2, FOXO/DAF-16, 14-3-3/ftt1/ftt2]

Running title: Ageing and stress regulation by 14-3-3 in C. elegans

Correspondence

Prof. Simon Galas, CRBM-CNRS, 1919 route de Mende, F34293 Montpellier cedex 5,

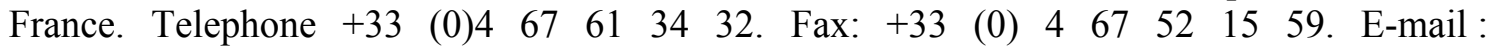
simon.galas@crbm.cnrs.fr 


\section{Abstract}

Caenorhabditis elegans life span, stress resistance and metabolism are regulated by the Insulin/IGF-1/DAF-2/DAF-16 pathway. DAF-16, a member of FOXO/Forkhead transcription factor family can be targeted by 14-3-3 proteins to promote stress resistance. We have identified a 14-3-3 C. elegans homolog which promotes life span by both DAF-2-dependent and -independent mechanisms and by an unexpected DAF-16 -independent mechanism. Our results demonstrate that $C$. elegans 14-3-3 proteins modulate stress responsive genes throughout adulthood. In conclusion, 14-3-3 can be considered as an acute stress-responsive regulator as well as a sustained modulator of the Insulin/IGF-1/DAF-2/DAF-16 regulatory pathway in promoting life expectancy of growing old worms. 


\section{Introduction}

Cell regulatory pathways, including signal transduction, apoptosis, stress response and transformation can be targeted by one or several 14-3-3 gene isoform products in organisms ranging from yeast to human (Van Hemert et al., 2001). The 14-3-3 family members are discrete binding proteins with no intrinsic enzymatic activity. However, the 14-3-3 proteins can act as enzymatic regulators as well as localization anchors or adaptator molecules with scaffold activity that can, in turn, stimulate various protein-protein interactions (Van Hemert et al., 2001). The Caenorhabditis elegans (C. elegans) genome encodes two 14-3-3 genes: $f t t-$ 1 and $f t t-2$ ( $f t$ for fourteen-three-three; Wang and Shakes, 1994). While a reported simultaneous expression of $f t t-1$ and $f t t-2$ during early embryogenesis may allow cooperative effects, the divergent expression patterns during pre-reproductive or adulthood stages argue for different respective biological functions (Wang and Shakes, 1997). Thus, while the germline enriched ftt-1 gene product (Wang and Shakes, 1997) - also known as par-5 - is involved in the asymmetric cortical localization of other par gene products that can, in turn, influence the general asymmetry determinism in early embryos (Morton et al., 2002), the ftt-2 gene expression pattern is largely non overlapping with the former because of a reported somatic expression that also takes place at later developmental stages (Wang and Shakes, 1997).

The expression pattern of a specific reporter transgene for $f t t-2$ and the $f t t-1$ further supports this view (Wang et al., 2006), altogether these reports make the ftt-2 gene product a bona fide candidate for a transduction pathway regulator during post-embryonic or post-developmental stages of C. elegans.

Mammalian FOXO homologs are well known targets of 14-3-3 proteins (Van Hermert et al., 2001; Brunet et al. 1999) but it has been shown that active C. elegans DAF-16 transcription factor, a FOXO family transcription factor (Lin et al., 1997; Ogg et al., 1997; Kenyon 2005), can be inhibited by 14-3-3 -dependent and -independent pathways in cell culture (Cahill et al. 2001). The conserved DAF-2/IGF-1 signalling pathway (Kenyon 2005) is believed to regulate life span through inhibition of the daf-16 gene product and it has been proposed that differential signalling may originate at the DAF-2/IGF-1 receptor to regulate daf-16 gene product activity leading to the emergence of different phenotypic daf-2 allele class mutants (Gems et al., 1998; Nanji et al., 2005). Moreover, the IGF-1 receptor itself, as well as downstream transducers such as 3-Phosphoinositide-dependent kinase-1 (PDK1) or IREs (insulin receptor substrates), are 14-3-3-reported targets (Van Hemert et al. 2001; Craparo et al., 1997; Sato et al., 2001). Recent reports demonstrated a positive worm life span modulation by 14-3-3 through a cooperative interaction mechanism involving both SIR-2.1 and FOXO/DAF-16: a mechanism fully suppressible by a daf-16 loss-of-function mutation or RNAi inactivation (Wang et al., 2006; Berdichevsky et al. 2006; Li et al., 2007). This new regulatory pathway has been linked to a stress-dependent mechanism acting in parallel to the DAF-2/IGF-1 signalling pathway to activate FOXO/DAF-16 and promote life span (Berdichevsky and Guarente 2006).

This report demonstrates that $C$. elegans 14-3-3 gene products can promote life span by both FOXO/daf-16 dependent and independent mechanisms. Furthermore we also reveal a specific daf-2 class mutant allele requirement for 14-3-3 genes to promote life span, together with different effects on physiological traits including both thermal and oxidative stress resistance, development, dauer arrest and fat storage. Finally, our work extends C. elegans 14-3-3 involvements in biological pathways functioning with acute stress response and sustained life span regulation. 


\section{Materials and Methods}

\section{Strains and worm cultures}

Worms were cultured and maintained as described previously (Brenner, 1974). We used the following strains: Wild type Bristol (N2), GR1307 daf-16(mgDf50), CB13710 daf-2(e1370), JT709 pdk-1(sa709), JT9609 pdk-1(sa680), GR1318 pdk-1(mg142), CF1330 muEx128 [pKL79 (daf-16a::GFP)+pRF4 rol-6 (su1006)], CL2070 dvIs70, CF1553 muIs84 [pAD76(sod-3::GFP), CL2166 dvIs19 [pAF15(gst-4::GFP::NLS)] which were all provided by the Caenorhabditis Genetics Center (funded by the NIH National Center for Research Resources). DR1569 daf-2(e1365) was kindly provided by Donald L. Riddle, University of British Columbia, Canada. Unless specified, worms were cultured at $20^{\circ} \mathrm{C}$ or $15^{\circ} \mathrm{C}$ for JT709 pdk-1(sa709).

\section{RNAi constructs}

The $f t t-1$ RNAi construct was obtained by subcloning a 709bp HindII/BgIII fragment from the pCP5 vector into the pPD129.36 (L4440) vector (a generous gift of Johnathan Ewbank, CNRS, Marseille, France) while ftt-2 RNAi construct was generated by subcloning a $531 \mathrm{bp}$ HindIII fragment from the pSE16 vecto into the pPD129.36 (L4440) vector. Both pCP5 and pSE16 ftt cDNA clones were a generous gift from Diane Shakes (College of William and Mary, Virginia, USA). After transformation with either pPD129.36, ftt-1 or ftt-2 RNAi contruct, HT115 (DE3) strain bacteria were grown in LB medium with both Ampicillin (50 $\mathrm{mg} / \mathrm{ml})$ and Tetracyclin $(12,5 \mathrm{mg} / \mathrm{ml})$ during eight hours, then seeded on NGM plates containing FUdR $(98,5 \mathrm{mg} / \mathrm{ml})$, Ampicillin $(50 \mathrm{mg} / \mathrm{ml})$ and IPTG $(1 \mathrm{mM})$ and induced overnight.

\section{RNAi feeding and lifespan assays}

Adult stage RNAi: L4 larvae fed with RNAi producing bacteria were allowed to grow on NGM plates at $20{ }^{\circ} \mathrm{C}$ until they died. To avoid offspring during experiments we used plates with FUdR (Mitchell et al., 1979). Dead worms were scored every day until they were all dead. Vitality was assessed by the animal response to one or several gentle nose touch. Worms that died by scrolling out of agar plates were scored as censored data. XLSTAT-life statistical software (Addinsoft, New York, NY, USA) was used to plot survival data by the Kaplan Meier method and differences between survival curves were calculated using the Log Rank test with $95 \%$ confidence.

Developmental stage RNAi and dauer assay : Five to ten L4/young adult hermaphrodites for each strain were permitted to egg-lay for two hours on plates seeded with transformed HT115 at $22,5^{\circ} \mathrm{C}$. The progeny was then allowed to grow on these same conditions. Delay to reach L4 stage was checked comparing to N2 on control RNAi conditions. All the worms growing to L4/young adult stage were transferred onto NGM plates with only FUdR and E. Coli OP50 strain and scored for life span trials, whereas dauer larvae were scored until around 72 hours and transferred onto fresh RNAi plates and observed until they left this stage and scored for dauer exit. For statistical analysis of the life span data curves we considered only the trials that gave a high percentage of dauer in parallel.

\section{$\underline{\text { Real-time PCR }}$}


Synchronised N2 adult worms were transferred on RNAi plates at $20^{\circ} \mathrm{C}$ during one day and then collected by washing with M9 buffer and pelleted. Total RNA isolation was done using TRIzol Reagent (Invitrogen) and RNA quantification was assessed by both gel electrophoresis and UV spectrophotometric analysis. cDNAs were synthesized with random hexamers by using Ready-To-Go ${ }^{\mathrm{TM}}$ You-Prime First-Strand Beads kit (GE Healthcare) according to the manufacturer's protocol. Real-time PCR reactions were performed using LightCycler Fast Start DNA Master ${ }^{\text {plus }}$ SYBR Green I kit in capillaries. Triplicates for each sample were included for one single reaction.

The real-time PCR primers for TBA2 are: Forward primer: 5'CCTCCTCCGAATGAATGAAA-3'; Reverse primer: 5'TCCGATACTGGAAACGGAAG3'. Primers for $f t t-1$ are: Forward primer: 5'-GTTTCAGAAGACTTGCTCAC-3'; Reverse primer: 5'-CTCGGTCACTTTCTTCATGGC-3'. Primers for ftt-2 are: Forward primer: 5'CAGTGACTCATCCACTTCAAGC-3'; 2 primer: 1 5'AACGCTCAGCCTGCTCGGCAAG-3'. All the PCR products were checked by sequencing. To avoid Light-Cycler cross amplification of RNA sequence included in RNAi-fed worms, both $f t t-1$ and $f t t-2$ specific forward primers were designed to hybridize the 5'-UTR region not included in the RNAi-targeted sequences. The corresponding reverse primers were designed to hybridize the boundary of the first and the second exons in order to prevent crossamplification of unspliced RNAs. $t b a-2$ was used as the internal control and the RNA level of each gene of interest was normalized to the tba-2 level for comparison. Real-time PCR reactions were performed three times using independent RNAi worms and RNA isolations and results were treated as described (Pfaffl, 2001). All of quantified PCR products in this study were previously sequenced for molecular identity verification.

\section{Nile red fat staining and handling of worms for transgene observations}

We used Nile Red (9-diethylamino-5H-benzo[a]phenoxa-]phenoxazine-5-one) obtained from Molecular Probes (N-1142) dissolved with acetone and mixed with transformed HT115 DE3 E. coli strain, at a final concentration of $0,5 \mu \mathrm{g} / \mathrm{ml}$. Five L4/young adult hermaphrodites of each strain were allowed to egg-lay for two hours at $22,5^{\circ} \mathrm{C}$ under RNAi conditions and then removed. The progeny was left on these plates until it reached L4/young adult stage. Nile red treatment resulted in uptake and incorporation of the dye into lipid droplets in intestinal cells, the main site of worm fat storage.

L4/young adults from either CF1553, CL2070 or CL2166 strains were transferred onto NGM plates prepared for RNAi feeding induction with FUdR. After four and eighteen days of treatment, sample worms were collected and observed under epifluorescence microscopy.

\section{Oxidative and heat shock stress resistance assays}

After one week of RNAi treatment at $20^{\circ} \mathrm{C}$ adult worms, were transferred onto 24-well plates (about 50 worms per well), with $10 \mathrm{mM}$ of Menadione (Sigma Aldrich) and HT115 bacterias mixed with S complete medium to maintain the RNAi treatment during the assay. Complete $\mathrm{S}$ medium mixed with HT115 bacteria was used as a control. Experiments were carried out for 7 days at $20^{\circ} \mathrm{C}$. We scored the number of alive and dead worms each day, using $1 \mu \mathrm{M}$ SYTOX Green Nucleic Acid Stain (Molecular Probes) to discriminate dead worms from alive ones. Survival histograms were analysed with XLSTAT software (Addinsoft, New York, NY, USA). For heat shock stress induction, adult worms fed on RNAi seeded plates at $20^{\circ} \mathrm{C}$ for one week were placed on a $37^{\circ} \mathrm{C}$ water bath for 2,5 hours then returned to $20^{\circ} \mathrm{C}$ and scored 
for dead worms.

\section{Microscopy observation and pixel quantification}

Microscopy observations were performed on a Zeiss AxioImager.Z1 with a Photometrics CoolSnap HQ2 camera at either 40X or 100X magnification with Zeiss Plan Neofluar-1.3 or Zeiss Apochromat-1.4 objectives respectively. Nile red staining was assessed with a Zeiss FS00, BP530-585, FT600, LP615 red filter while GFP signal was assessed with a Zeiss GFP FS38HE, BP470/40, FT495, BPJ25/50 GFP filter. Pixel quantification was performed with Metamorph v7.1 software (Molecular Devices, USA) while data analysis by a student t-test was done using the XLSTAT software (Addinsoft, New York, NY, USA). 


\title{
Experimental Results
}

\author{
ftt-2 gene but not $f t t-1$ gene modulates life span in Daf-16 loss-of-function genetic \\ background.
}

We used RNA-mediated gene interference (RNAi) to reduce the expression of either $f t t-1$ or ftt-2 C. elegans genes in wild type worms (hereafter denoted as N2). We verified the the specificity of our RNAi constructs by quantifying ftt-1 and ftt-2 RNA level in N2 RNAi worms by real-time PCR (see materials and methods). As shown in Fig. 1, we reported both significant and specific gene knock down of both ftt-1 or ftt-2 mRNA expression in worms fed with the corresponding RNAi bacteria. Morevover, similar to previous reports (Morton et al., 2002; Wang et al., 2006; Berdichevsky et al., 2006) using par-5 mutants or ftt-1 RNAi, we observed almost $100 \%$ dead embryos in the progeny of adults with $f t t-1$ RNAi.

In contrast, the ftt-2 RNAi treatment didn't reveal any embryonic lethality. As previously reported (Berdichevsky et al., 2006), we observed that ftt-1 and ftt-2 RNAi treatment induced DAF-16::GFP nuclear localization (data not shown). Additionally, $f t t-2$ RNAi during larval stage induced a low percentage of "bagging" phenotype (15\% for wild type worms), as a result of internal hatching of the progeny and a small fraction ( $8 \%$ for wild type worms) of protruding vulvae phenotype in adults. No "bagging" phenotype was observed with $f t t-2$ RNAi during adulthood. Therefore, the gene specificity of the RNAi treatment correlated with phenotypic traits.

We then assessed for 14-3-3-dependent effects on worm life span by subjecting N2 adults to either ftt-1 or ftt-2 RNAi. As shown in Fig. 2.A and Table 1, N2 worms showed a significant life span shortening while $f t t-1$ RNAi yielded an intermediate life span shortening compared to $f t t-2$ RNAi. This result gives an indication that $\mathrm{N} 2$ worms are more prone to $f t t-2$ for life span modulation than to $f t t-1$ when maintained in stress-free conditions since all of these experiments where conducted at $20^{\circ} \mathrm{C}$.

The DAF-16 transcription factor is believed to be situated at the major crossroads of the various regulatory pathways that modulates life span and stress resistance in C. elegans (Lin et al., 1997; Ogg et al., 1997; Kenyon 2005; Dillin et al., 2002). Recent reports (Wang et al., 2006; Berdichevsky et al., 2006; Li et al., 2007) demonstrated new 14-3-3 regulations extending stress resistance and life span in a DAF-16 dependent manner. However, active DAF-16 inhibition by 14-3-3 dependent and independent pathways in cell culture has been reported (Cahill et al., 2001). To clarify the relationship between 14-3-3 and DAF-16dependent life span modulation mechanisms, we assessed life span modulation in a daf16( $m g D f 50)$ deletion mutant allele treated with $f t t$ RNAi. As shown in Fig. 2.B and Table 1, we noticed a highly significant reduction in life span upon $f t t-2$ RNAi. In contrast ftt-1 RNAi did not induce any significant life span reduction. This unexpected result demonstrates, for the first time, that DAF-16-dependent and -independent mechanisms can be targeted by 14-33 to promote life span. Moreover, because life span modulation by $f t t-1$ is fully suppressible by a $d a f-16$ loss-of-function mutation, we propose that $d a f-16$ is the only target of $f t t-1$ for life span regulation in worms while $f t$ - 2 is able to modulate life span by an as yet unknown daf16-independent mechanism together with a daf-16-dependent process.

Previous studies have already reported the RNAi potency to specifically target a given $\mathrm{ftt}$ gene product in worm (Morton et al., 2002; Berdichevsky et al., 2006; Wang et al., 2006; Li et al., 2007). Therefore, the data presented here strongly support that RNAi contructs can specifically target the corresponding $f t t-1$ or $f t-2$ gene products without any cross-phenotypic consequences. 

background.

Major biological outputs of the DAF-2/IGF-1 pathway are believed to elicit FOXO/DAF-16 activity modulation since strong loss-of-function mutations in the daf-16 gene can suppress the induced phenotypes by point mutation in the DAF-2/IGF-1 receptor tyrosine kinase (Ogg et al., 1997; Lin et al., 2001). We therefore assessed 14-3-3 life span modulation effects in the $d a f-2(e 1365)$ and $d a f-2(e 1370)$ reduction-of-function mutant because they belong respectively to daf-2 class 1 and class 2 phenotypic mutants (Gems et al., 1998). Amino-acid substitution (A580T) in the ligand-binding domain of daf-2(e1365) and in the tyrosin kinase receptor domain (P1465S) of daf-2(e1370) have been proposed to explain the phenotypic difference between both $d a f-2$ class mutants (Gems et al., 1998; Nanji et al., 2005). Different daf-2 class mutants and corresponding phenotypic traits are believed to result from the mobilisation of different signalling intermediates that originate at the DAF-2/IGF-1 receptor and result in a subset of common and different downstream unknown complex processes, that can in turn, converge to regulate the daf-16 gene product (Gems et al., 1998; Nanji et al., 2005). We expected these experiments to be highly informative with respect to 14-3-3 dependent regulations on worm life span by providing new insights into 14-3-3-dependent phenotypic traits expressing or not a daf-2 class mutant requirement to be revealed. As shown in Fig. 2.C,D and Table 1, both $f t t-1$ and $f t t-2$ RNAi treatments induced a significant life span reduction when applied to both daf-2 class mutants. However, as attested by the $50^{\text {th }}$ percentile (see Table 1), the strongest life span reduction was observed upon ftt-2 RNAi treatment on both $d a f-2$ class mutants. Moreover, relevant statistical difference between both $f t t-1$ and $f t-2$ RNAi treatment was only evident on daf-2(e1370) and we were also surprised to note a return to a wild type mean life span of the daf-2(e1370) worms (compare mean life span of experiments code $\mathrm{A}$ and $\mathrm{M}$ on Table 1) upon ftt-2 RNAi treatment only. Altogether, these observations indicate that daf-2(e1370) class 2 mutant is more prone to ftt-2 -dependent life span modulation than daf-2(e1365) class 1 mutant.

It has been reported that DAF-16 activity regulation mainly operates by the 3Phosphoinositide-dependent kinase-1 /AGE-1 signalling pathway in daf-2(e1370) class 2 rather than in daf-2(e1365) class 1 mutants (Gems et al., 1998; Nanji et al., 2005). Moreover, 3-Phosphoinositide-dependent kinase-1 (PDK1) has been reported to be negatively targeted in its activity by 14-3-3 (Sato et al., 2001). Our results are in agreement with the former observation if we consider that $f t-2$ may act by the same pathway in C. elegans. If 14-3-3 does so, we then expect to observe a pronounced life span shortening by ftt-2 RNAi in a daf$2(e 1370)$ class 2 mutant background because such effect may result in a slight rise in DAF-16 inhibition through a loss of 14-3-3-dependent negative regulation acting on PDK1. The results described above argue for such epistasis interaction. Moreover, if $p d k-1$ also represents the main target for $f t t-2$ regulation, one might expect to observe an unmodified life span of $p d k-1$ knock out worms upon $f t$ - 2 RNAi while, on the other hand, the $f t t-1$ RNAi should do so. We then submitted the $p d k-1$ (sa709) loss-of-function allele to RNAi and, as expected (Fig. 2. E), the ftt-2 RNAi failed to affect life span significantly ( $\mathrm{P}=0.796$; Table 1) while ftt-1 RNAi remained with a statisticaly significant $(\mathrm{P}=0.0001$; Table 1$)$ life span modulation activity. This result argues for the fact that $f t t-2$ dependent life span modulation operates mainly towards the $p d k-1$ gene, a result fully correlated with the observed life span modulation induced by $f t t-2$ RNAi in the daf- 2 class 2 mutant. However, $f t t-1$ RNAi produced a negative modulation on life span during the same experiment (Fig. 2. E), while we previously reported an opposite effect in both daf-2 class mutant (Fig. 2. C, D). It is possible that such ftt-1 dependent negative modulation on life span, also independent from the $p d k-1$ component of the DAF- 
2/DAF-16 pathway, was previously undetectable in both daf-2 class-mutant where the strongest life span modulation is observed.

In an attempt to specify either $f t t-1$ or $f t t-2$ respective life span effects throughout PDK-1 signaling modulation, we next assessed a $p d k$ - $1(\mathrm{mg} 142)$ gain-of-funtion mutant for RNAi knock down experiment. As shown in Figure 2. F, the ftt-2 RNAi but not the ftt-1 RNAi induced a significant life span modulation in comparizon with control. This result is relevant to a negative modulation of $p d k-1$ activity by $f t t-2$ that can be highlighted here by a life span shortening effect upon RNAi. The absence of a significant modulation effect on life span by $f t-1$ in the same genetic background also reinforces the view that ftt-1 doesn't regulate life span towards $p d k-1$ in the DAF-2/DAF-16 regulatory pathway (Fig. 2. F and table 1) and also indicate that $f t$ - 1 negative modulation on life span is also masked in the $p d k$ - 1 gain-offunction background as observed below for both daf-2 class mutant.

Thus, we show here for the first time a $d a f-2$ class mutant-dependent modulation of 14-3-3 on C. elegans life span regulation. These results indicate that 14-3-3 influences longevity not only when DAF-2 activity is high and DAF-16 low (as in N2), but also when DAF-2 activity is low and DAF-16 high (as in daf-2 mutants). Therefore, while ftt-1 dependent life span modulation is restricted to $\mathrm{N} 2$ and only one of both $d a f-2$ class mutants, the $t t-2$ dependent life span modulations can affect $\mathrm{N} 2$ and both daf-2 class mutants assessed, as well as a daf-16 loss-of-function background. This observation argues for the predicted $f t t-2$ gene function on life span modulation previously defined on the basis of its expression pattern (Morton et al., 2002; Wang et al., 2006) in comparison with the one of ftt-1. Moreover, on the basis of respective modulation of life span reported here with ftt RNAi on daf-2 class mutants, we present here further evidence arguing that $f t$ - 2 is a more potent life span modulator than $f t-1$. Altogether, our results indicate that $f t t-2$ can modulate worm life span with different order of magnitude depending on the DAF-2/DAF-16 transduction level targeted that can be summarized for by a strong modulation effect when transduction levels targeted lie between $\underline{d a f-2 \text { and } p d k-1 \text { then intermediate effect when transduction targeted levels are between } p d k-1}$ and $d a f-16$ and discrete modulation effect by a daf-16 independent mechanism.

\section{ftt-2 modulates intrinsic worm thermotolerance.}

Daf-2 reduction-of-function mutants show an increased intrinsic thermo tolerance (itt) phenotype (Lithgow et al., 1995) and conversely, heat shock treatment can induce rapid nuclear localization of DAF-16 (Henderson and Johnson 2001; Henderson et al. 2006). Because this itt phenotype trait is believed to require DAF-16 activity (Lin et al., 2001) we then assessed for $f t t-1$ and $f t-2$ involvement in this process. As shown in Fig. $\underline{3}$.A, no thermal resistance difference between $\mathrm{N} 2$ worms fed with either control RNAi or $f t t-2$ RNAi was noted while we observed a slight and time limited $(\mathrm{P}=0.05)$ significative resistance difference when ftt-1 RNAi. On the opposite, daf-2(e1370) or daf-2(e1365) worms subjected to same treatment after either $f t t-1$ or $f t t-2$ RNAi (Fig. 3. B and C) were less resistant. As attested by the level of significance observed $(\mathrm{p}=0.005)$, a protracted effect on daf-2(e1370) thermal stress resistance was observable upon $f t t-2$ RNAi while a delayed but strongest effect was noted when $f t t-1$ RNAi (Fig. B). A comparable modulation by both $f t-1$ and $f t t-2$ RNAi on thermal stress resistance was observed for daf-2(e1365) genetic background (Fig. 3. C). This observation may indicate, as we reported for life span, that intrinsic thermotolerance of both $d a f-2$ class mutant allele worms are prone to ftt-dependent regulation than wild type. More interestingly, the rise in thermal stress sensitivity when daf-2 mutant worms were fed with $f t t-2$ RNAi was not observable when daf-16( $\mathrm{mgDf50})$ mutant or $p d k-1$ (sa680) loss of function mutant (Fig. 3. E) where fed by the same (Fig. 3. D). Moreover, identical result was 
noted upon ftt-1 RNAi (Fig. 3. D and E). This observation indicates that the itt trait phenotype is a part of the stress resistance mechanism allowing $f t t-2$ and, to a lesser extend $f t t-1$, to specifically promote worm life span throughout DAF-16 dependent mechanism as described here and in Berdichevsky et al. (2006). The lack of ftt-2 RNAi effect on N2 worms upon thermic stress may be due to a threshold sensibility difference in comparison with daf-2 mutants, which are also regarded as constitutively stressed worms, thereby explaining, as described (Berdichevsky et al., 2006), the higher sensitivity of stress resistant mechanisms to 14-3-3-dependent regulation. However, these results are in agreement with the expected itt phenotype for the corresponding genetic backgrounds tested, because daf-16(mgDf50) appeared less heat-resistant than wild type or $d a f-2(e 1370)$ mutant worms in control RNAi conditions.

\section{ftt-2 can modulate various stress responses.}

DAF-2/IGF-1 pathway regulates stress resistance in worm (Gems et al., 1998) and treating a daf-2(e1370) mutant with daf-16 RNAi during the reproductive period can also modulate its life span (Dillin et al., 2002). DAF-16 gene products are believed to act as major cellular defense regulators when worms are facing stress. However, while some stress responsive genes, as for example sod-3 (Henderson et al., 2006; Honda and Honda 1999; Murphy et al., 2003; Lee et al., 2003b; McElwee et al., 2003; Oh et al., 2006), are mainly FOXO/DAF-16 targets, other stress-inducible genes, such as heat-shock proteins (HSPs), can be synergistically regulated by FOXO/DAF-16 and HSF-1 transcription factors (Hsu et al., 2003; Morley and Morimoto 2004). Moreover, it has been demonstrated that DAF-16 is essential for maximal $h s p-16$ expression and for life span extension conferred by $h s p-16$ over expression (Walker and Lithgow 2003). Glutathione $S$-transferases (GSTs), oxidative stress responsive genes products, are believed to participate in metabolisation of cell compounds by posttranslational glutathione tripeptide conjugation. Moreover, GSTs have already been reported to be up-regulated in daf-2 mutant (McElwee et al., 2006) and by various physical or ROSinducer chemical stressors (Leiers et al., 2003). We therefore decided to test for stress responsive gene modulation during adulthood of ftt-2 RNAi-induced worms because it had already been reported (Dillin et al., 2002) that DAF-2/IGF-1 pathway could influence ageing and stress resistance during ageing. To this aim, we used a GFP reporter which had been used to assay for DAF-16 activity: The sod-3::GFP (Berdichevsky et al., 2006; Libina et al., 2003), as well as a HSPs reporter: $h s p-16::$ GFP (Link et al., 2002) and the oxidative stress-inducible reporter transgene gst-4::GFP (Leiers et al., 2003; Link et al., 2002) whose constitutive or ROS-induced expression is not dependent on DAF-16 activity (Henderson et al., 2006). As shown in Fig. 4, while the expression level of these GFP reporters does not appear to change between adulthood day four to day eighteen (compare Fig. $\underline{4}$.A and B; Fig. $\underline{4}$.E and F; Fig. $\underline{4}$.I and $\mathrm{J}$ ) in control worms, the expression pattern becomes greatly modified during the same adulthood interval when worms are fed with $f t t-2$ RNAi (compare Fig. 4 .C and D; Fig. 4.G and H; Fig. $4 . \mathrm{K}$ and L). However, we can clearly discriminate two groups of GFP reporter genes from which induction response to $f t t-2$ RNAi treatment differs. One first group with a high expression induction level (sod-3::GFP and gst-4::GFP) and a second with a limited induction level ( $h s p-16:: \mathrm{GFP})$. To increase discriminative analysis of reporter transgenes, we decided to perform pixel quantification analysis for both successive groups of growing old worms (see Material and Methods). As shown in Fig. 4.M, we detected a gradual rise in the expression level for each transgene during ageing and, as expected, to a lesser extent for $h s p$ 16::GFP transgene. Moreover, the limited $h s p-16::$ GFP rise in expression is also notable when we compare both adulthood periods tested. By contrast, we noted (particularly at day eighteen of adulthood) an important change for sod-3::GFP reporter expression level while gst-4::GFP 
showed an intermediate level of induction. Because these experiments were conducted in a stress-free condition, we conclude that stress-responsive GFP transgenes have been tested for their basal ftt-2-dependent regulation during normal aging. This observation sheds new light onto the regulatory links between the DAF-2/IGF-1 pathway - Believed to influence ageing and stress resistance in growing old worms by a daf-16 dependent mechanism - and sod$3::$ GFP gene activation. However, we reported here that $h s p-16::$ GFP, another stress inducible gene, behaves differently during the same recorded adulthood time, from sod-3::GFP. This observation may indicate that $h s p-16$ gene expression regulatory mechanisms are less prone to $f t$ - 2 regulatory activity in an environment devoid of stress. Moreover, these results are in complete agreement with our previous experiments showing a lack of involvement of 14-3$3 / f t$-2 for itt phenotypic trait in wild type worms. Altogether, our observations argue for a sustained involvement of $\mathrm{ftt}$-2 gene product for stress-responsive gene regulation during adulthood as reported for the DAF-2/IGF-1/DAF-16 pathway. Moreover the interesting observation of a pronounced sod-3::GFP trangene induction at day eighteen in comparison with day four upon treatment with $f t$-2 RNAi (compare Fig. 4.C and D) may also point out that regulatory mechanisms operating to maintain the physiological status of growing old worms are not the same during the whole post-developmental period. A recent report (Henderson et al., 2006) introduced that oxidative stress could induce nuclear translocation of DAF-16 without any detectable over-expression of sod-3::GFP reporter. However, our experiments clearly demonstrate a strict relationship between nuclear DAF-16 translocation and sod-3::GFP reporter induction in non-stress induction conditions although upon $f t t-2$ RNAi.

\section{ftt-2 modulates worm oxidative stress resistance by a $d a f-16$-independent mechanism.}

The DAF-2/DAF-16 regulatory pathway is believed to link worm oxidative stress resistance to life span (Finkel and Holbrook 2000; Hsu et al., 2003). We therefore investigated the effect of Menadione in different genetic backgrounds, believed to affect worm oxidative stress resistance. The Menadione (2-methyl-1,4-naphthoquinone or vitamin K3) is a lipid-soluble substance that has been extensively used as an oxidant stressor in yeast (Shackelford et al., 2000) as well as in Arabidopsis thaliana (Baxter et al., 2007). Menadione can generate reactive superoxide ions, which can be further oxidized to yield hydrogen peroxide and has been reported to deplete total glutathione concentration in yeast cells by generation of menadione S-glutathione conjugates (Zadzinski et al., 1998).

We examined the effect of Menadione by determining survival of adult worms subjected to $10 \mathrm{mM}$ Menadione after one week of either $\mathrm{ftt}-1$ or $\mathrm{ftt}-2$ RNAi treatment (see Material and Methods). As expected in control conditions, the daf-16(mgDf50) mutant was less resistant (Fig. 5. D) to Menadione treatment than N2 (Fig. 5. A), which also appeared less resistant to both daf-2(e1370) and daf-2(e1365) reduction-of-function mutants (Fig. 5. B and C). Moreover, we observed that daf-16 mutant life span was significantly reduced $(\mathrm{P}=0.005)$ upon both ftt RNAi in comparison with control RNAi (Fig. 5. D) while the same treatment did not induced a significant survival difference in either $\mathrm{N} 2$ or daf-2(e1370) genetic backgrounds (Fig. 5. A and B). However, we observed a time limited significant effect of ftt-1 RNAi on daf-2(e1365) loss of function mutants survival (Fig. 5. C). Altogether, our results indicate that both $\mathrm{ftt}-1$ and $\mathrm{ftt}-2$ are involved in promoting Menadione oxidative stress resistance by acting through a daf-16-independent pathway. Although the mechanism through which $f t t-1$ and $f t t-2$ promotes daf-16(mgDf50) stress resistance is yet unknown, it is possible that different mechanism operates because we previously demonstrated that ftt-2 is the only 14-3-3 ortholog able to promote life span in both daf-16 loss-of-function and daf-2 reduction-of-function 
genetic backgrounds. Altogether, these findings show that $f t t-2$ can determinate stress resistance even in a non-stress resistant genetic background such as daf-16(mgDf50).

\section{Dauer formation can be enhanced by ftt-2 inactivation.}

Several $d a f-2$ thermosensitive $(t s)$ reduction-of-function mutations can result in constitutive dauer larval formation: a quiescent (ie. a non obligatory stress resistant arrested stage that differs from obligatory diapause stage affecting other worm species) larval stage with developmental arrest, non-feeding activity, stress resistance and long-lived characteristics (Klass M. and Hirsh D., 1976). Dauer induction fold seems to depend on daf-2ts class alleles that can be summarised as more severe for class 2 and weaker for class 1 mutant. Therefore, the semi-restrictive temperature $\left(22,5^{\circ} \mathrm{C}\right)$ applied to daf-2ts mutants can modify the population that constitutively enters the dauer stage (Gems et al., 1998). We believed that such semi-restrictive temperature would help us to reveal possible $f t t-1$ or $f t$-2-dependent dauer formation process by generating a facilitated constitutive dauer commitment subthreshold. We therefore assessed relevant class 2 daf-2(e1370) and class 1 daf-2(e1365) allele for dauer formation upon $f t t-2 \mathrm{RNAi}$ at $22.5^{\circ} \mathrm{C}$.

While $f t t-1$ RNAi produced no detectable effect on dauer formation in all genetic backgrounds tested (Fig. 6), we observed a highly significant difference ( $\mathrm{P}=0.005)$ upon $\mathrm{ftt}-2 \mathrm{RNAi}$ on $d a f-$ 2 class 1 mutant and a less significant difference $(\mathrm{P}=0.05)$ on daf- 2 class 2 mutant (Fig. 6). This observation indicates that class 1 daf-2ts mutant alleles are more prone to $f t t-2$ dependent regulation than class 2 for dauer commitment while we reported the opposite effect for life span assays. This observation indicates for the first time the existence of a daf-2 class mutant allele-specific modulation for dauer formation by $f t$ genes. However, because dauer entry and exit are not equivalent physiological processes, as revealed by time course microarray analysis (Wang and Kim 2003), we next asked if dauer exit might behave with the same difference between both daf-2 mutants alleles tested. We then checked dauer population and observed full dauer exit for both daf-2 reduction-of-function allele populations 120 hours after hatching.

\section{ftt-2 influences worm life span by acting during development.}

While daf-2 RNAi can influence aging when applied only during worm adulthood (Kenyon 2005; Dillin et al., 2002), daf-2 deletion mutants are affected by non-conditional arrest at embryonic or first larval stage (Gems et al., 1998). In addition, genes encoding mitochondrial respiratory chain components have been reported to influence worm life span if reduction-offunction, mimicked by RNAi, was applied only before the reproductive stage (Dillin et al., 2002b; Lee et al., 2003a).

We observed a variation on the worm populations that did not arrest at dauer stage upon either $f t-1$ or $f t-2$ RNAi at semi-restrictive temperature. Thus, while $82 \%$ of $d a f-2(e 1370)$ worms did not arrest at dauer stage on control RNAi (see Material and Methods), the corresponding rate decreased to $59 \%$ and reached up to $\underline{81 \%}$ when worms were fed for $f t t-2$ and $f t t-1$ RNAi respectively.

Moreover, in the daf-2(e1365) genetic background, non-dauer arrested worms fraction reached $83 \%$ on control RNAi but only $39 \%$ on $f t-2$ RNAi while ftt- 1 RNAi induced a comparable rate to wild type as $80 \%$. Wild type worms did not revealed any dauer formation on control or on both RNAi treatments at $22.5^{\circ} \mathrm{C}$ and $25^{\circ} \mathrm{C}$ too (data not shown).

We then assessed the worm fraction with uninterrupted larval stage progression for developmental rate when RNAi knock down. Both wild type and daf-16(mgDf50) worms fed by $f t$ t- 2 or $f t-1$ RNAi protracted 24 hours to reach the fourth larval stage ( 96 hours after hatching) when compared to control worms. Moreover, both daf-2(e1370) and daf-2(e1365) 
worms protracted 48 hours to reach the fourth larval stage (120 hours after hatching) when ftt2 RNAi but only 24 hours (96 hours after hatching) when ftt-1 RNAi. This observation indicates that $f t t-2$, and to a lesser extend $f t t-1$, reduction-of-function applied during worm development can induce a "slow living phenotype" by perhaps acting, more or less directly, through the modulation of a yet unknown transduction pathway able to induce others defects as reported here and in previous reports (Wang et al., 2006; Berdichevsky et al., 2006).

Because development was affected with the same order of magnitude on both daf-2 class mutant at semi-restrictive temperature, we then addressed for a possible influence of this developmental modulation on subsequent post-developmental stages.

To this aim, worms that reached the fourth larval stage were shifted to $f t t-2$ or $f t t-1$ RNAi free condition at $22.5^{\circ} \mathrm{C}$ and assessed for life span (see Material and Methods). As shown in Fig. 7, we observed a highly significant $(\mathrm{P}=0.001)$ life span shortening for wild type worms induced with $f t t-2$ RNAi than ftt-1 RNAi in comparison with controls (Fig. 7.A and Table 2). Moreover, both daf-2(e1370) and daf-2(e1365) worms showed a remarkable life span shortening on both RNAi (Fig. 7. C and D and Table 2) in comparison with non-induced worms and wild type worms after equivalent treatment (Table 2). This unexpected result highlights for the first time that ftt-2, and to a lesser extend ftt-1, RNAi knock down can shorten life span if administered only during development in both wild type and daf-2 reduction-of-function mutants.

Moreover, the observed higher mortality rate for both $d a f-2$ class mutants indicates that they are more prone than wild type worms to $\mathrm{ft} t$ dependent regulations for development - A similar observation is also reported below for post-developmental life span experiments (Fig. 2; Table1).

However, because $d a f-16(m g D f 50)$ worms behaved the same as both daf-2 mutants (Fig.7. B), we also conclude that $f t$-dependent control of development involves both DAF-2/DAF-16dependent and -independent regulatory mechanisms.

The high mortality rate and mortality curve shape observed during our experiments may reflect possible non-specific sickness acting on life span by ftt RNAi knock down but other observations argue against such a simple conclusion. Thus, as shown in Table 2, it is possible to observe that ftt RNAi do not impair by the same worms life span with different genetic backgrounds. For example, while the $f t t-2$ RNAi induced a $26 \%$ mean life span reduction in wild type, we showed a here $60 \%$ mean life span reduction for daf-2(e1365) and up to $67 \%$ reduction for $d a f-2(e 1370)$ while $d a f-16(m g D f 50)$ worms where less affected with a $44 \%$ mean life span reduction only.

If $f t$ RNAi knock downs produce a non-specific effect on life span, one might expect a proportional linear rise in life span reduction from short-living worms (as daf-16(mgDf50)) to long-living worms (daf-2 class mutants). However, this was not the case because short-living worms were more affected than wild type worms. Moreover, the daf-2 class 2 (daf-2(e1370)) life span was more affected upon ftt-2 RNAi than daf-2 class 1 (daf-2(e1365)) as already reported for post-developmental life span experiments below (Figure 2, table 1) were daf2(e1370) and daf-2(e1365) life span were reduced by $36 \%$ and $32 \%$ respectively.

This observation provides new insight into both $f t$ - 2 and $f t t-1$-dependent regulations on life span that need to be specifically assessed during development or adulthood to avoid missinterpretation of results due to possible unrelated cumulative effects produced by $f t$ RNAi administered during pre- and post-reproductive periods. For example, while we reported both a small percentage of protruding vulvae phenotype (15\%) and bagging phenotype in adult worms fed on OP50 bacteria after ftt-2 RNAi induced during development, we noted that the same $f t t-2$ RNAi treatment applied during adulthood induced only the life span reduction phenotype. Therefore, noticing that $d a f-2$ mutants life expectancy was more affected than the 
wild type clearly indicates that $f t-2$, and to a lesser extend $f t t-1$, acts as a more potent modulator during development when DAF-2 signalling is low and DAF-16 activity high.

\section{C. elegans fat storage can be modulated by ftt-2.}

During our dauer commitment analysis we noted a reproducible "dark appearance" shape variation between control and ftt-2 RNAi induced worms. Such "dark appearance" shape phenotype can be explained by lipid droplet accumulation as already described for daf-2 reduction-of-function mutation or dauer larvae (Kimura et al., 1997).

The increased fat content trait of $d a f-2$ mutants has been shown to be fully suppressible by daf-16 mutants (Lin et al., 1997; Ogg et al., 1997) and we reported below (Fig.6) that ftt-2 but not ftt-1 RNAi can increase dauer larvae (a well characterized resistant stage with high lipid accumulation) commitment in two different daf-2 class mutants together with a more significant effect for class 1 mutant allele. Moreover, it has been demonstrated that some daf2 mutations (class 2) can induce dauer-like trait expression in adults while others (class 1) do not and show a normal metabolism instead (Gems et al., 1998). However, recent microarray analysis reported the identification of a transcriptional products group whose expression was highly similar in both dauers and daf-2 mutant adults (McElwee et al., 2003; McElwee et al., 2006) while some other groups behaved differently. We therefore assessed if $f t-2$ or $f t t-1$ could influence lipid metabolism in both $d a f-2$ class mutant alleles with the same order of difference as for dauer commitment. As shown in Fig. 8 (A-L), the apparent fat content of daf-16(mgDf50): Fig. 8.D and F, and daf-2(e1365): Fig. 8.G and I was reduced upon ftt-2 RNAi while both wild type worms (Fig. 8.A and C) and daf-2(e1370) fat content (Fig. 8.J and L) appeared less affected. Nevertheless, the ftt-1 RNAi did not induce apparent lightening in lipid staining excepted for $d a f-2(e 1370)$ genetic background (Fig.8. J and K).

Therefore, relative quantification (see Material and Methods) of Nile Red lipid staining (Fig. 9) provided more insight with respect to the difference of fat storage that appeared more significantly impaired in daf-2(e1365) and daf-16(mgDf50) worms than in daf-2(e1370) and N2 worms when $f t$ - 2 RNAi while $f t t-1$ RNAi produced a significan lipid staning reduction in daf-2(e1370) worms only.

This observation provides novel insights into the striking opposite effect of $f t t-2$ RNAi on both dauer induction and its accompanying lipid droplet accumulation trait. More unexpectedly, we also found a significant fall of lipid droplet accumulation in a dauer defective genetic background mutant as daf-16(mgDf50). On the basis of this experimental evidence, we propose that $f t-2$ can promote lipid accumulation by a $d a f-16$-independent pathway. Therefore, $f t t-2$ can increase dauer commitment in daf-2 class 1 mutant allele, an observation indicating a modulation via alternative regulatory pathway that may (Gems et al., 1998; Nanji et al., 2005) or may not emerge from the DAF-2 receptor. Thus, while both dauers and $d a f-2$ adults contain large quantities of lipid droplets, it has been reported (Walker and Lithgow 2003) that triglyceride synthesis enzymes are not expressed in the same fashion during these two $C$. elegans life history stages leaving open possible differences in the regulatory pathways targeted by $f t t-2$ besides of DAF-2/IGF-1 regulatory pathway. Moreover, a quantitative mass spectrometry analysis reported (Dong et al., 2007) a subset of significant abundant proteins in daf-2(e1370) mutant were able to either slow down or accelerate ageing rate when targeted by RNAi knock down then living open the possibility for compensatory mechanism with opposites effects on the rate of ageing but altogether with a positive global effect as observed in long living daf-2 mutants. It is possible that some molecular targets of ftt-2 may fall into both classes of protein thereby explaing opposite effects on dauer commitment and lipid accumulation reported in this study. 


\section{Discussion}

The $f t t-2$ can act as a life span determinant regulator in $C$. elegans.

Because of a reported lethal phenotype for both $f t t-1$ and $f t$ - 2 loss-of-function mutants (Wang et al., 2006; Berdichevsky et al., 2006; Li et al., 2007), we used RNAi in an attempt to investigate the $C$. elegans 14-3-3 genes homolog involvement in life span regulation. The RNAi experiments described in our study allowed us to analyze developmental as well as post-developmental ftt-dependent modulation of various $C$. elegans life history traits.

We report here that $f t t-2$ is a more potent life span modulator in all of the $C$. elegans genetic backgrounds that we assessed as well as in a closer worm species such as $C$. briggsae (Araiz and Galas; manuscript in preparation). As previously proposed in other reports (Cahill et al., 2001; Berdichevsky et al., 2006), the $C$. elegans ftt genes can promote cytoplasmic retention of phosphorylated DAF-16 but can also mediate activation of a 14-3-3/SIR-2.1/DAF-16 stress mediated-dependent pathway to elicit stress resistance and life span extension. Here we show that $f t t-2$ can promote $C$. elegans life span in stress-free conditions. Moreover, we show for the fist time the existence of two actions levels of $f t t-2$ (as well as for $f t t-1$ ) for life span modulation. Thus, while ftt-2 gene knockout by RNAi can induce side morphological problems (protruding vulva or bagging phenotype, as also reported elsewhere (Wang et al., 2006; Berdichevsky et al., 2006)), together with a strong life span shortening, the same RNAi induction only during the post-developmental period induces a highly significant life span shortening without morphological side effects. We also demonstrate here the specificity of $f t t-$ 1 and $f t$ - 2 RNAi induction by the observation of non-overlapping induced phenotypes.

In summary we show here that $f t t-2$, and to a lesser extent the $f t t-1$, can positively modulate the life expectancy during worm adulthood by acting at developmental stages only. This mechanism may reflect the 14-3-3 pleiotropic effects on worm signalling systems because both daf-2 class mutant assessed in our RNAi knock down experiments appeared to be modulated to the same relative extent difference as the control. We also report in this work that $\mathrm{ftt}-2$ can promote worm life span throughout a negative modulation of the $p d k-1$ while $\mathrm{ftt}$ 1 does not. However, we show here that daf-2 class 2 mutants are more prone to ftt-2 modulation for life span than daf-2 class 1 mutant alleles during adulthood. Previous reports (Gems et al., 1998; Nanji et al., 2005) have shown that daf-2 class 2 mutant alleles are genetically characterised by a greater loss of PI3kinase signalling, which reflects multiple signal outputs from the DAF-2 receptor. It is possible to conclude that main effect on life span by $f t t-2$ is mediated by this transduction pathway (as depicted in Fig. 9) because of a $p d k-1$ requirement as revealed by our experiments. Altogether, the experiments described here demonstrate for the first time a $f t t-2$ (as well as $f t-1$ ) life span different modulation in both daf-2 class 1 and class 2 mutants.

\section{ftt-2 can modulate life span in a daf-16 null genetic background}

In this report, we show that daf-16 loss-of-function mutant retains a ftt-2 but not $f t$ - 1 dependent life span modulation (Fig. 9). This result indicates that additional mechanisms can regulate worm life span in a daf-16-independent but ftt-2-dependent manner. Moreover, we show for both daf-2 class mutants assessed in this work, that ftt-2 RNAi only during developmental life history period can also exert a deleterious effect on worm life span.

Previous reports introduced that SIR-2.1, a C. elegans NAD-dependent deacetylase homolog, can interact with FTT-1 and FTT-2 proteins to activate DAF-16 and extend the life span of stressed worms only (Wang et al., 2006; Berdichevsky et al., 2006; Li et al., 2007 and Fig. 8). Moreover, these reports showed that sir-2.1 or $f t t-1$ and $f t-2$ were not required for life span 
extension when reduced DAF-2/IGF-1 signalling although such studies only assessed one daf2 class mutant. We demonstrate here the existence of a new regulatory mechanism involving the $f t t-2$ gene and acting via daf-16 and the DAF-2/IGF-1 pathway together with another yet unknown daf-16 -independent process for normal life span modulation, stress resistance and metabolism regulation (Fig. 8). Because previous studies did not assess for the FTT-2/Sir2.1/DAF-16 stoechiometric regulatory complex and because it is always possible that cytoplasmic retention of DAF-16 upon $f t t-2$ RNAi does not reflect the fate of the whole DAF16 cellular pool, this new ftt-2 target pathway may use part of the FTT-2 protein pool to regulate life span in a daf-16 -independent manner.

\section{Dauer formation and lipid accumulation can be modulated by ftt-2 knock down.}

It has been reported that dauer formation regulation of $d a f-2$ class 1 mutant occurs mainly via a parallel pathway from the daf-2/age-1/daf-16 pathway (Gems et al., 1998; Nanji et al., 2005). However we show here that dauer commitment of both daf-2 class mutants are greatly enhanced when $f t t-2$ is targeted by RNAi while $f t t-1$ RNAi produces no detectable effect.

Because we show that daf-2 class 2 allele mutants are more prone to positive life span regulation by ftt-2 than class 1 mutant allele and because we show that dauer induction upon $f t t-2$ RNAi can be greatly enhanced in $d a f-2$ class 1 mutant, we propose that an alternative daf-2/daf-16 pathway is more prone to $f t t-2$ regulation for dauer formation but not for life span modulation. Moreover, because lipid droplet accumulation is a well reported accompanying metabolic phenotype of worm dauer induction as well as in dauer constitutive phenotypes, we demonstrate here that $f t-2$ must be unexpectedly involved in promoting lipid droplet accumulation together with a negative influence in dauer commitment while $f t t-1$ is not involved in dauer commitment regulation but only for lipid accumulation in daf-2 class 2 mutant.

\section{ftt-2 can modulate worm stress resistance responses during adulthood period.}

We reveal here that $f t t-2$ promotes stress resistance by both daf-2 dependent and daf-16 independent processes. We also show that this effect is correlated with the up-regulation of a subset of daf-16 target genes that have been involved in stress resistance. However, because our experiments were done in stress-free conditions, we can conclude here for a positive modulation by $f t$ - 2 in promoting stress-responsive genes. Moreover, we also demonstrate that $f t-2$ gene can promote the up-regulation of DAF-16 target genes that have been involved in stress resistance. We also demonstrate by two independent observations (heat shock assay and HSP-16::GFP expression) that heat shock responsive gene expression is not modulated by ftt2 thereby providing new insights into the fine tuning regulations of DAF-16 molecular targets. Such fine tuned regulations may use the new emerging family of FOXO/DAF-16 cofactors that can account for simultaneous daf-16 -dependent and -independent processes regulating life span programs in stress conditions in C. elegans (Berdichevsky and Guarente 2006).

In conclusion, this study demonstrates, for the first time, the ability of the C. elegans 14-3-3 genes to modulate the life expectancy during adulthood even in a stress-free environment.

The new modulation properties on the various physiological traits assessed in this work (Fig. 9) yet extend the involvement of 14-3-3-dependent regulations from short time events, such as the one counteracting the danger of sudden darkness in plants (Mackintosh 2004) or acute stress response in $C$. elegans to a more sustained event such as normal life span regulation. 


\section{Acknowledgments}

We thank Theresa Stiernagle and the Caenorhabditis elegans Genetics Center for providing strains. We are grateful to Donald L. Riddle for the daf-2(e1365) strain and Diane Shake for kindly providing us with the 14-3-3/ftt cDNAs clones. We are especially grateful to MRI (www.mri.cnrs.fr) and its technical assistance. We thank Julia Pfitzner, Victoria Malchin, Nga Nguyen and Professor Ulrich Mok, from Lise-Meitner-Oberstufenzentrum, Berlin, Germany, for technical assistance. We thank Dr. May Morris and Dr. Jean Michel Bellanger and Dr. Andrew Burgess for critical reading of the manuscript. We thank Dr. Anne Blangy for helpful with qPCR experiments. This work was supported by the CNRS, C. Araiz. is a PhD fellow of AFM (www.afm-france.org) and ARC (www.arc.asso.fr/). 


\section{References}

Baxter CJ, Redestig H, Schauer N, Repsilber D, Patil KR, Nielsen J, Selbig J, Liu J, Fernie AR, Sweetlove LJ. (2007) The Metabolic Response of Heterotrophic Arabidopsis Cells to Oxidative Stress. Plant Physiol. 143, 312-325.

Berdichevsky A, Viswanathan M, Horvitz H.R. and Guarente L. (2006) C. elegans SIR-2.2 interacts with 14-3-3 Proteins to activate DAF-16 and extend life span. Cell, 125, 1165-1177.

Berdichevsky A, Guarente L. (2006) A stress response pathway involving sirtuins, forkheads and 14-3-3 proteins. Cell Cycle. 5, 2588-2591.

Brenner, S. (1974). The genetics of Caenorhabditis elegans. Genetics 77, 71-94.

Brunet A, Bonni A, Zigmond MJ, Lin MZ, Juo P, Hu LS, Anderson MJ, Arden KC, Blenis J, Greenberg ME. (1999) Akt promotes cell survival by phosphorylating and inhibiting a

Forkhead transcription factor. Cell. 96, 857-868.

Cahill CM, Tzivion G, Nasrin N, Ogg S, Dore J, Ruvkun G and Alexander-Bridges M. (2001)

Phosphatidylinositol 3-Kinase signaling inhibits DAF-16 DNA Binding and function via 143-3-dependent and 14-3-3- independent pathways. J. Biol. Chem., 276, 13402-13410.

Craparo A, Freund R and Gustafson TA. (1997). 14-3-3 (epsilon) interacts with the insulinlike growth factor I receptor ans insulin receptor substrate in a phospho-serine dependent manner. J. Biol. Chem., 272, 11663-11669.

Dillin A, Crawford DK, Kenyon C. (2002) Timing requirements for insulin/IGF-1 signaling

in C. elegans. Science. 298, 830-834.

Dillin A, Hsu AL, Arantes-Oliveira N, Lehrer-Graiwer J, Hsin H, Fraser AG, Kamath RS, Ahringer J, Kenyon C. (2002b) Rates of behavior and aging specified by mitochondrial function during development. Science. 298, 2398-2401.

Finkel T, Holbrook NJ. (2000) Oxidants, oxidative stress and the biology of ageing. Nature. 408, 239-247.

Gems D, Sutton AJ, Sundermeyer ML, Larson PL, Albert PS, King KV, Edgley M and Riddle DL (1998) Two pleiotropic classes of daf-2 mutation affect larval arrest, adult behavior, reproduction an longevity in Caenorhabditis elegans. Genetics, 150, 129-155.

Henderson ST and Johnson TE (2001) daf-16 integrates developmental and environnemental inputs to mediate aging in the nematode Caeonrhabditis elegans. Curr. Biol., 11, 1975-1980. Henderson ST, Bonafe M, Johnson TE. (2006) daf-16 protects the nematode Caenorhabditis elegans during food deprivation. J Gerontol A Biol Sci Med Sci. 61, 444-4460.

Honda Y, Honda S. (1999) The daf-2 gene network for longevity regulates oxidative stress resistance and Mn-superoxide dismutase gene expression in Caenorhabditis elegans. FASEB J. 13, 1385-1393.

Hsu AL, Murphy CT, Kenyon C. (2003) Regulation of aging and age-related disease by DAF16 and heat-shock factor. Science. 300, 1142-1145.

Kenyon C. (2005) The plasticity of aging: insights from long-lived mutants. Cell. 120, 449460.

Kimura KD, Tissenbaum HA, Liu Y, Ruvkun G. (1997) daf-2, an insulin receptor-like gene that regulates longevity and diapause in Caenorhabditis elegans. Science, 277, 942-946.

Klass M, Hirsh D. (1976) Non-ageing developmental variant of Caenorhabditis elegans.

Nature. 260, 523-525.

Lee SS, Lee RY, Fraser AG, Kamath RS, Ahringer J, Ruvkun G. (2003a) A systematic RNAi screen identifies a critical role for mitochondria in C. elegans longevity. Nat Genet. 33, 40-48. Lee SS, Kennedy S, Tolonen AC, Ruvkun G (2003b) DAF-16 target genes that control C. elegans life-span and metabolism. Science. 300, 644-647. 
Leiers B, Kampkotter A, Grevelding CG, Link CD, Johnson TE, Henkle-Duhrsen K (2003) stress-responsive glutathione S-transferase confers resistance to oxidative stress in Caenorhabditis elegans. Free Radic Biol Med. 34, 1405-1415.

Libina N, Berman JR, Kenyon C (2003) Tissue-specific activities of C. elegans DAF-16 in the regulation of lifespan. Cell. 115, 489-502.

Dong MQ, Venable JD, Au N, Xu T, Park SK, Cociorva D, Johnson JR, Dillin A, Yates JR 3rd. (2007) Quantitative mass spectrometry identifies insulin signaling targets in C. elegans. Science. 317, 660-663.

Li J, Tewari M, Vidal M and Lee SS (2007) The 14-3-3 protein FTT-2 regulates DAF-16 in Caenorhabditis elegans. Dev Biol. 301, 82-91.

Lin K, Dorman JB, Rodan A, Kenyon C (1997) daf-16: An HNF-3/forkhead family member that can function to double the life-span of Caenorhabditis elegans. Science. 278, 1319-1322. Lin K, Hsin H, Libina N and Kenyon C (2001) Regulation of the Caenorhabditis elegans longevity protein DAF-16 by insulin/IGF-1 and germline signaling., Nat. Genet. 28: 139-145. Link CD and Johnson CJ (2002) Reporter transgenes for study of oxidant stress in Caenorhabditis elegans. Methods Enzymol. 353, 497-505

Lithgow GJ, White TM, Melov S, Johnson TE (1995) Thermotolerance and extended lifespan conferred by single-gene mutations and induced by thermal stress. Proc Natl Acad Sci USA. 92, 7540-7544.

Mackintosh C (2004) Dynamic interactions between 14-3-3 proteins and phosphoproteins regulate diverse cellular processes. Biochem J. 381, 329-342.

McElwee J, Bubb K, Thomas JH (2003) Transcriptional outputs of the Caenorhabditis elegans forkhead protein DAF-16. Aging Cell. 2, 111-121.

McElwee JJ, Schuster E, Blanc E, Thornton J, Gems D (2006) Diapause-associated metabolic traits reiterated in long-lived daf-2 mutants in the nematode Caenorhabditis elegans. Mech Ageing Dev. 127, 458-472.

Mitchell DH, Stiles JW, Santelli J, Sanadi DR (1979) Synchronous growth and aging of Caenorhabditis elegans in the presence of fluorodeoxyuridine. J Gerontol. 34, 28-36.

Morley JF, Morimoto RI. (2004) Regulation of longevity in Caenorhabditis elegans by heat shock factor and molecular chaperones. Mol Biol Cell. 15, 657-664.

Morton DG, Shakes DC, Nugent S, Dichoso D, Wang W, Golden A, Kemphues KJ. (2002) The Caenorhabditis elegans par-5 gene encodes a 14-3-3 protein required for cellular asymmetry in the early embryo. Dev Biol. 241, 47-58.

Murphy CT, McCarroll SA, Bargmann CI, Fraser A, Kamath RS, Ahringer J, Li H, Kenyon C. (2003) Genes that act downstream of DAF-16 to influence the lifespan of Caenorhabditis elegans. Nature. 424, 277-283.

Nanji M, Hopper NA and Gems D. (2005) LET-60 modulates effects of insulin/IGF-1 signaling on development and aging in Caenorhabditis elegans. Aging Cell. 4, 235-245. Oh SW, Mukhopadhyay A, Dixit BL, Raha T, Green MR, Tissenbaum HA. (2006) Identification of direct DAF-16 targets controlling longevity, metabolism and diapause by chromatin immunoprecipitation. Nat Genet. 38, 251-257.

Ogg S, Paradis S, Gottlieb S, Patterson GI, Lee L, Tissenbaum HA, Ruvkun G. (1997) The Fork head transcription factor DAF-16 transduces insulin-like metabolic and longevity signals in C. elegans. Nature. 389, 994-999.

Pfaffl MW. (2001) A new mathematical model for relative quantification in real-time RTPCR. Nucl. Acid. Res., 29:e45.

Sato S, Fujita N and Tsuruo T (2001) Regulation of kinase activity of 3_Phosphoinositidedependent Protein Kinase-1 by binding to 14-3-3. J. Biol. Chem., 277, 39360-39367.

Shackelford RE, Kaufmann WK, Paules RS (2000) Oxidative stress and cell cycle checkpoint function. Free Radic Biol Med. 28, 1387-404. 
Van Hemert MJ, Steensma HY, Van Heusden GP (2001) 14-3-3 proteins: key regulators of cell division, signalling and apoptosis. Bioessays. 23, 936-946.

Walker GA, Lithgow GJ. (2003) Lifespan extension in C. elegans by a molecular chaperone dependent upon insulin-like signals. Aging Cell. 2, 131-139.

Wang W, Shakes DC.(1997) Expression patterns and transcript processing of ftt-1 and ftt-2, two C. elegans 14-3-3 homologues. J Mol Biol. 268, 619-630.

Wang W, Shakes DC. (1994) Isolation and sequence analysis of a Caenorhabditis elegans cDNA which encodes a 14-3-3 homologue. Gene. 147, 215-218.

Wang J, Kim SK (2003) Global analysis of dauer gene expression in Caenorhabditis elegans. Development. 130, 1621-1634

Wang Y, Wook Oh S, Deplancke B, Luo J. Walhout AJM and Tissenbaum H A (2006) C. elegans 14-3-3 proteins regulates life span and interact with SIR-2.1 and DAF-16/FOXO. Mech Ageing Dev. 127, 741-747.

Zadzinski R, Fortuniak A, Bilinski T, Grey M and Bartosz G (1998) Menadione toxicity in Saccharomyces cerevisiae cells: activation by conjugation with glutathione. Biochem Mol Biol Int. 44, 747-759. 


\section{Table Legends}

Table 1 : Life span modulation by 14-3-3 depends on $C$. elegans genotype in stress-free conditions.

The tabulated data show the average results of four to eight independent RNAi trials. Worms of indicated genotypes were assayed for life span by feeding on the E. coli HT115 strain that express either $f t t-1, f t-2$ RNAi or control vector (see Material and Methods). XLSTAT-life statistical software (Addinsoft, New York, NY, USA) was used to plot survival data by the Kaplan Meier method and differences between survival curves were calculated using the LogRank test with $95 \%$ confidence.

(£) Represents the 50th percentile (the age when the survival fraction of animals reaches $0,50 \%$.

(€) Experiment identification code.

(\$) Probability of being identical to other life span experiment indicated in parentheses.

(*) Total death scored (number of censored values).

Table 2 : C. elegans life span shortening by pre-reproductive ftt-2 RNAi induction.

The tabulated data show the average results of four to five independent RNAi trials at $22.5^{\circ} \mathrm{C}$. Worms of indicated genotypes were fed on E. coli HT115 strain bacteria expressing either ftt2, ftt-1 or control RNAi during pre-reproductive developmental period then transferred to RNAi-free conditions and assayed for life span (see Material and Methods).

XLSTAT-life statistical software (Addinsoft, New York, NY, USA) was used to plot survival data by the Kaplan Meier method and differences between survival curves were calculated using the LogRank test with 95\% significance.

(@) Mean life span values (Standard Deviation).

(\$) Probability of being identical to other life span experiment indicated in parentheses.

(*) Total death scored (number of censored values).

\section{Legends to Figures:}

Figure 1 . The $f t t-1$ and $f t t-2$ RNAi treatments induce specific mRNA knock down. $\mathrm{N} 2$ were treated by RNAi feeding for either $f t t-1$ or $f t t-2$ gene specific knock down during adulthood (see materials and Methods). The y-axis indicates the relative RNA levels of either $f t t-1$ or $f t t-2$ normalized to the RNA level of tubulin $(t b a-2)$. One representative experiment with the standard error of the mean (S.E.M.) is shown. The $P$-value of $<0.005$ (**) $^{*}$ was determined by Student's $t$-test.

Figure 2. Both $C$. elegans 14-3-3 can shorten adult life span but only ftt-2 can do it in various genetic backgrounds.

Adult worm life-span analysis were perfomed on RNAi plates as described in Material and Methods. (A- $\underline{\text { F }}$ ) The squared lines represent the life span of animals of indicated genotype fed by bacteria carrying control RNAi vector. Circle lines and triangle lines represent life spans of indicated genotypes fed with bacteria carrying either the $f t t-2$ or the $f t t-1$ RNAi constructs. 
(A) Both $f t t-2$ and $f t-1$ RNAi shorten life span of wild type (N2) worms but with different order of magnitude.

(B) Only ftt-2 RNAi shortens life span in a daf-16 null genetic background.

(C-D) Only ftt-2 RNAi can shorten life span in both daf-2 class 1 and class 2 reduction-offunction mutants.

(E-F) The ftt-2 life span modulation operates mainly throughout $p d k-1$ gene.

See Table 1 for corresponding quantitative and statistical analysis. All experiments were performed at $20^{\circ} \mathrm{C}$.

Figure 3. $f t$-2 RNAi knock down shortens worm survival after heat shock induction in daf-2 reduction-of-function background but not in both wild type (N2) or daf-16 loss-offunction genetic background.

(A-E) Young adults of the indicated genotype were fed by bacteria carrying either $f t t-1, f t t-2$ or control RNAi contruct, then subjected to heat shock (Material and Methods) and their viability scored at the indicated times. Results presented are mean values +/- S.E.M. of five independent experiments. Statistical analysis was done by a Student's $t$-test where ${ }^{*} P$ indicates $P<0.05$ signification level. All experiments were performed at $20^{\circ} \mathrm{C}$.

Figure 4. ftt-2 RNAi can promotes activation of stress responsive gene reporters during adulthood in non-stressing conditions.

(A-L) Wild type (N2) young adult worms carrying either a sod-3::GFP (A-D), a $h s p-16:: \mathrm{GFP}$ $(\mathrm{E}-\mathrm{H})$ or a gst-4::GFP (I-L) stress reporter transgene were fed by bacteria carrying either control RNAi vector (A-B; E-F; I-J) or ftt-2 RNAi contruct (C-D; G-H; K-L) and scored for transgene expression at fourth $(\mathrm{A}, \mathrm{C}, \mathrm{E}, \mathrm{G}, \mathrm{I}, \mathrm{K})$ and eighteenth days $(\mathrm{B}, \mathrm{D}, \mathrm{F}, \mathrm{H}, \mathrm{J}, \mathrm{L})$ of adulthood by fluorescence microscopy as described in Material and Methods. Note higher level of sod$3:$ GFP transgene expression at day eighteen of adulthood only when worms fed $f t-2$ RNAi contruct (D) in comparison with same worms scored at fourth day of adulthood (C) or with worms fed on control RNAi at both days of adulthood (A-B). Both sod-3::GFP (C-D) and gst4::GFP (K-L) transgene inductions during adulthood are greater than $h s p-16:: \mathrm{GFP}(\mathrm{G}-\mathrm{H})$ when compared to controls at both adulthood periods (sod-3::GFP: A-B; $h s p-16:: \mathrm{GFP}$ : E-F; gst-4::GFP: I-J). All images were taken at 40X magnification using fluorescence microscopy with an exposure time of $1000 \mathrm{~ms}$ for A-D; $200 \mathrm{~ms}$ for E-H and $1000 \mathrm{~ms}$ for I-L. All experiments were performed at $20^{\circ} \mathrm{C}$. the positive transgene induction control tests are not shown. (M) $\mathrm{ftt}-2$ RNAi-dependent induction of stress responsive genes during adulthood. Wild type (N2) young adult worms carrying either sod-3::GFP, $h s p-16::$ GFP or a gst-4::GFP stress reporter transgene were fed by bacteria carrying either control RNAi vector or $f t t-2$ RNAi construct and scored for stress responsive transgene expression at fourth and eighteenth days of adulthood. Quantifications were done by fluorescence microscopy as described in Material and Methods on at least ten animals. Bars represent the standard error (SEM) and mean values are expressed after normalisation with respective control RNAi, which represent $100 \%$. Statistical Student's $t$-test $(* P$ value $<0.05 ; * * P$ value $<0.0005)$ comparison between day four and day eighteen values are: ${ }^{* *} P=0.0003$ for sod-3::GFP; $P=0.09$ for $h s p$ $16:: \mathrm{GFP}$ and $* P=0.01$ for $g s t-4:: \mathrm{GFP}$. All experiments were performed at $20^{\circ} \mathrm{C}$.

Figure 5. Both ftt RNAi knock down prevent oxidative stress resistance by a daf-16 independent mechanism.

(A-D) Young adults of the indicated genotype were fed by bacteria carrying either $f t t-1$, ftt-2 or control RNAi contruct, then subjected to oxidative treatment by $10 \mathrm{mM}$ Menadione (as described in Material and Methods) and their viability scored at the indicated time. Results presented are mean values +/- S.E.M. of at least five independent experiments. Statistical 
analysis was done by a Student t-test at $* P<0.05$ and $* * P<0.0005$ signification level. The total number of worms scored for each genotype and RNAi condition are in parentheses: Wild type Control RNAi (N=218); Wild type-ftt-1 RNAi ( $\mathrm{N}=174)$; Wild type-ftt-2 RNAi ( $=238)$; daf-16(mgDf50)-Control RNAi (N=169); daf-16(mgDf50)-ftt-1 RNAi (N=587); daf16(mgDf50)-ftt-2 RNAi (N=180); daf-2(e1365)-Control RNAi (N=159); daf-2(e1365)-ftt-1 RNAi ( $\mathrm{N}=164)$; daf-2(e1365)-ftt-2 RNAi ( $\mathrm{N}=183)$; daf-2(e1370)-Control RNAi ( $=219)$; daf-2(e1370)-ftt-1 RNAi ( $=277)$; daf-2(e1370)-ftt-2 RNAi (N=193). All experiments were performed at $20^{\circ} \mathrm{C}$.

Figure 6. ftt-2 RNAi knock down promotes dauer constitutive induction phenotype in both daf-2 class 1 and 2 reduction-of-function mutants.

Worms of indicated genotype were allowed to hatch on bacteria carryings either $f t-1, f t t-2$ or control RNAi contruct at $22.5^{\circ} \mathrm{C}$ in order to create a dauer constitutive phenotype induction subthresold. Dauer larval arrest was scored as described in Material and Methods. The total number of worms scored for each genotype and RNAi condition are in parentheses: Wild type Control RNAi (N=469); Wild type-ftt-1 RNAi ( $\mathrm{N}=\underline{130})$; Wild type-ftt-2 RNAi (N=475); daf2(e1370)-Control RNAi (N=1219); daf-2(e1370)-ftt-1 RNAi (N=480); daf-2(e1370)-ftt-2 RNAi (N=1052); daf-2(e1365)-Control RNAi ( $\mathrm{N}=1138)$; daf-2(e1365)-ftt-1 RNAi ( $=\underline{621})$; daf-2(e1365)-ftt-2 RNAi ( $\mathrm{N}=1235)$. Results presented are mean values +/- S.E.M of at least five independent experiments. Statistical student's $t$-test was used to compare datas from control and $f t t$ RNAi fed worms and give either $* P<0.05$ and $* * P<0.0005$ thresold signification level.

Figure 7. Reducing ftt-2 by RNAi during development leads to apparent sickness at subsequent post-developmental stage.

Worms of indicated genotype were allowed to hatch on bacteria carryings either ftt-1, ftt-2 or control RNAi. Worm population that did not arrest as dauers was fed on bacteria carrying either control RNAi vector or $f t t-1$ or $f t t-2$ RNAi until they reached young adult stage then transfered on new plates and fed by classical OP50 E. coli strain then scored for life span. Shortening life span in all of genetic backgrounds was also characterised by sickness defects such bagging or protruding vulvae phenotye. See text for details and Table 2 for corresponding quantitative and statistical datas analysis. All of the experiments were conducted at $22.5^{\circ} \mathrm{C}$.

Figure 8. ftt-2 can positively modulate lipid droplets reserves.

(A-L) Worms of indicated genotype were allowed to hatch on bacteria carrying either control RNAi vector, $f t t-1$ or $f t t-2 \mathrm{RNAi}$ at $22.5^{\circ} \mathrm{C}$ together with Nile Red lipid staining until young adult stage. Pictures were taken at $40 \mathrm{X}$ magnification for $200 \mathrm{~ms}$ by fluorescence miscroscopy as described in Material and Methods. (M) Lipids droplets quantification reveals a highly significant reduction of lipid content in daf-16(mgDf50) loss-of-function mutant upon ftt-2 RNAi knock down only. Quantifications of Nile Red lipid staining were done by fluorescence microscopy as described in Material and Methods on at least ten animals. Bars represent the standard error (SEM) and mean values are expressed after normalisation with respective control RNAi, which represent $100 \%$. Statistical Student's $t$-test comparison between $\mathrm{ftt}$ RNAi and respective control where $* P$ value $<0.05$ and $* * P$ value $<0.005$, give the following results for $f t t-2: * P=0.02$ for Wild type; $* P=0.03$ for $d a f-2(e 1370) ; * P=0.009$ for daf-2(e1365); $* P=0.05$ for daf-16(mgDf50) and for ftt-1: $P=0.26$ for Wild type; $* * P=$ $\underline{0.0009 \text { for } d a f-2(e 1370) ; P=0.18 \text { for } d a f-2(e 1365) ; P=0.77 \text { for } d a f-16(m g D f 50)}$. 
Figure 9. A model depicting the involvement of 14-3-3 in DAF-16-dependent and independent mechanims regulating normal life span and acute stress response in $C$. elegans.

We propose a two component model in which 14-3-3 can modulate (A) no stress and (B) stress dependent physiological responses.

(A) In non-stress conditions, 14-3-3 is permanently at work to modulate physiological traits in a DAF-2-dependent manner and we show that PDK1 is a target for 14-3-3 regulation. We demonstrate in this work that 14-3-3 can also modulate physiological traits by acting independently of DAF-16.

(B) In stress conditions, we showed that 14-3-3 promotes oxidative stress resistance in a DAF-16-independent manner. On the contrary, our work demonstrates that 14-3-3 can modulate thermotolerance by a DAF-2/DAF-16-dependent mechanisms introducing a new side regulatory stress responsive pathway element to the one already described by Berdichevsky et al., 2006. 
Reviewer \#1:

The authors conducted a fairly detailed analysis of the role of 14.3.3 or fourteen-three-three (ftt-1 and ftt-2) in specifying life span and stress resistance in the nematode $\mathrm{C}$. elegans. The data presented in the paper are of good relevance to the field of the journal even though they are not fully conclusive and the author interpretation of their result is not very straightforward. The authors showed that ftt-2 RNAi significantly shorten C. elegans life span while ftt-1 RNAi yielded an intermediate life span shortening when compare to ftt-2 RNAi. With classical epistatic experiment they then studied the role of 14.3.3 in the insulin/IGF/daf-2 pathway, the major pathway controlling $\mathrm{C}$. elegans longevity. Interestingly they concluded that 14.3.3 regulate life span by both DAF-16-dependent and independent mechanisms. Namely that DAF-16 is the solely target of ftt- 1 for life span specification while ftt-2 regulated life span both dependently and independently of daf16.

The authors then explore the role ftt- 1 and $\mathrm{ftt}-2$ in the longevity specification of $\mathrm{s}$ different classes of daf-2 mutantsdaf-2 mutants: class 1 or daf-2 (e1365) and class 2 or daf-2 (e1370). They suggested that despite both ftt-1 and ftt-2 RNAi induced a significant life span reduction when applied to both class of mutants, ftt- 2 is a more potent modulator of life span than ftt-1 especially in the daf-2 (e1370) class 2 worms. These conclusions do not seem to be strongly supported by their results and are indeed mostly speculative. Yet, based on these observations, the author continue the rest of the work only analyzing ftt-2 effect on different phenotypes (stress resistance, dauer formation) in the context of the daf-2 pathway.

\section{Authors answer: Please note that all modifications have been underlined in the revised manuscript.}

\section{MAJOR POINTS}

1) To better map the role of 14.3 .3 in the regulation of longevity, stress resistance, dauer formation and lipid accumulation, it would have been interesting to also test the effect of ftt-1 RNAi in the different experiments carried out in the paper. Do the author have any data on ftt1 other than life span analysis? These data would add more complexity to the paper and probably make it even more speculative on the mechanisms by which 14.3.3 specify the above phenotypes, yet they would give a more complete analysis on the role of 14.3.3 in the specification of phenotypes regulated by the daf-2/daf-16 pathway.

\section{1) Authors answer:}

To increase the comparative analysis of $f t t-1$ and $f t t-2$ effect on $C$. elegans, new experimental datas using $f t t-1$ RNAi knock out have been included in the manuscript as for thermotolerance, oxidative stress resistance, dauer committment and fat storage. Thank's to this important comment on our manuscript, we where able to highlight new differences in modulatory effects between $f t t-1$ and $f t t-2$ on dauer formation as well as lipid accumulation.

2) One of the argument used to support the conclusion that ftt-2 is a more potent modulator of life span than ftt-1 especially in the daf-2 (e1370) class 2 worms is that, besides their life span outcome, DAF-16 activity regulation mostly operates by PI3K/AGE-1 signaling pathway in daf-2 (e1370) class 2 mutant rather than in daf-2 (e1365) class 1 . Moreover, previous observations showed that the PI3K/PDK-1 is negatively regulated by fft- 2 . Given that, mostly based on these notions, the rest of the work only analyzed ftt- 2 effect on different phenotypes in the context of the daf-2 pathway, it would be informative to analyze the effect of both ftt-1 and $\mathrm{ftt}-2 \mathrm{RNAi}$ in a pdk-1 knock out strain. Indeed if the author suggestion that ftt-2 mainly 
regulates longevity in the class 2 daf-2 (e1370) pathway is correct, ftt-2 RNAi might not affect the increase in life span of the pdk-1 knock out while on the other hand ftt-1 should do so. The pdk-1 knock out strain is available from the CGC and the experiment would add strong support for the author hypothesis that daf- 2 class 2 of mutants is more prone to $\mathrm{ftt}-2$ life span regulation through PDK-1.

2) Authors answer:

Our initial manuscript hypothetised that $f t t-2$ is a potent longevity modulator because of the observed strong life span effect upon RNAi knock out in the daf-2(e1370) class 2 mutant background. We then hypothetized that ftt-2 may modulate longevity by acting through PDK-1 - a PI3K/AGE-1 signaling pathway component - because of a previously reported negative modulation of PDK-1 by 14-3-3 in human cell culture (Sato et al., 2001).

As proposed for our manuscript revision, we have included new ftt-1 and ftt-2 RNAi knock out experiments on $p d k$ loss-of-function/gain-of-function mutant alleles. These new data are in accordance with the original hypothesis and show that ftt-2 RNAi do not increase life span in a pdk-1 loss of function gentic background while ftt-1 do.

\section{MINOR POINTS}

1) Page 4, lane 18: vecto should be vector

2) Page 7, end of lane 24: the author should add something after $<$ the same $>$ in the sentence

"...14.3.3 /ftt-2 may act by the same PATHWAY (?) ..."

3) Page 7, lane 49: daf-2(e1370) or daf-2(e1365): the order should be inverted daf-2(e1365) or daf-2(e1370)

Minor points authors answer : All of the minor points below have been corrected in this revised manuscript. 
Reviewer \#2: This work focuses on the roles of two 14-3-3 family members in C. elegans life span and stress resistance with particular focus on the Insulin/IGF-1/DAF-2/DAF-16 pathway.

Authors answer: Please note that all modifications have been underlined in the revised manuscript.

14-3-3 proteins form a large family of approximately $30 \mathrm{kDa}$ acidic proteins that exist primarily as homo- and heterodimers within eukaryotic cells. They play roles in diverse signal transduction pathways. In this study the authors use RNAi to inhibit expression of two 14-3-3 family members, encoded by the ftt- 1 and ftt- 2 genes. The effects of ftt- 1 and ftt- 2 RNAi on life span, stress resistance, development and fat deposition are monitored in wild-type C. elegans, and in daf- 2 and daf-16 mutants. The results presented are interesting and relevant and suggest that the 14-3-3 family members modulate stress-responsive gene expression, and life span, and that this modulation is both dependent and independent of the DAF-2/DAF-16 pathway.

There are several concerns that should be addressed:

1. The RNAi constructs are described on page 4. The authors have not addressed the potential for cross-talk between the two RNAi constructs. That is, does feeding RNAi to ftt-1 impact RNA levels of just ftt-1 and not ftt-2? It is important to document that the RNAi feeding impacts the desired target, as well as provide an indication of the specificity of the constructs. This is important because the authors assert that there are distinct actions of ftt- 1 and ftt- 2 on life span, stress response, etc.. For example, is the life span decrease mediated by ftt-2 RNAi due to effects solely on $\mathrm{ftt}-2$ RNA?

1) Authors answer:

Our work reports distinct actions of ftt-1 and ftt-2 RNAi knock down on physiological traits as for longevity, stress response, lipid accumulation and dauer commitment as well. However, in its original form, our manuscript do not show a molecular evidence for both specificity and impact of RNAi knock down on mRNA level for both ftt-1 and ftt-2. We have included quantitative PCR in the reviser form of our manuscript that can be seen on figure 1 and described on material and methods section. The corresponding references have been added in the bibliography section.

2. One of the conclusions is that the ftt-2 RNAi shortens life span of both types of daf-2 mutants (Class I, a ligand binding domain mutant, and Class II, a kinase mutant), while in contrast ftt-1 RNAi shortens life span of only one of the daf- 2 mutants. However, the data presented in Figure 1C and D seem to suggest that ftt-1 RNAi shortens life span of both types of daf- 2 mutants. The interpretation that ftt- 2 is the "most important player" affects subsequent experiments. For example, the thermotolerance assays presented in Figure 2 focus exclusively on $\mathrm{ftt}-2$.

2) Authors answer :

We agree both referee remarks about the failure of our initial manuscript to show a comparative analysis of the ftt- 1 and ftt-2 effects on all the physiological traits assessed for. We have thus included new experimental data about ftt-1 RNAi effects on all physiological traits that were only assessed for $\mathrm{ftt}-2$ in the original manuscript. This new data argue for a more wider effects of ftt- 2 than $\mathrm{ftt}-1$ on $\mathrm{C}$. elegans physiology as for lipid accumulation, dauer induction and longevity as well. This last point is discussed in the results section to improve the demonstration. Moreover, an error on the last lane of Table 1 has been fixed yet and allow the reader to clearly discriminate that while it is 
possible to show a significant longevity difference $(P<0.0001)$ between $\mathrm{ftt}-1$ and $\mathrm{ftt}-2$ RNAi in daf-2(e1370) background, no significant difference $(P=0.623)$ is observed when equivalent RNAi treatment in daf-2(e1365). This difference in the modulation effect of both ftt RNAi implies that :

1- ftt-2 RNAi knock down always induces the larger life span reduction in all genetic backgrounds assessed for. The population scale that we also assessed for argues for the data robustness;

2- the significant ftt-2 RNAi effect on longevity in daf-2(e1370) genetic background is also explained by the additional data presented in the figure 2 showing a clear pdk-1 dependent effect for ftt-2 life span modulation as hypothesized in our initial manuscript.

3. Feeding of RNAi during development is used to argue that the 14-3-3 family members play important roles during development, and that their inhibition during the development window can shorten adult life span (Figure 6). However, the authors acknowledge that the life span curves indicate that this might be due to "non-specific sickness". It seems difficult to conclude that a developmental window is important for 14-3-3 effects on adult life span, if the deficiency of the factor leads to a "sick" adult animal.

3) Authors answer:

We have re-examined the data and find out a non-linearity between ftt RNAi and life expectancy of various genetic background assessed for. This observation is discussed in the results section and the corresponding figure number has been incremented to Figure 7 because of quantitative PCR addition on a new Figure 1.

Minor comments:

The manuscript must be edited for grammar, spelling and clarity of sentence structure. On page 10 several "track changes" notations in word are still present. The text of the Discussion refers to Figure 9, (instead of the final Figure 8).

Authors answer :

We have edited the manuscript as proposed and the figure number changed according to the new revised manuscript and the new figure 1 that we added. 


\begin{tabular}{|c|c|c|c|c|c|c|c|}
\hline Strain & RNAi & $\begin{array}{c}\text { 50th } \\
\text { percentile } \\
(£) \\
\end{array}$ & $\begin{array}{c}\text { Mean life span } \\
\text { days (+ /-SD) }\end{array}$ & Max life span & $\begin{array}{l}\text { code } \\
\text { (Ū) }\end{array}$ & $\begin{array}{c}\text { Statistics } \\
\text { (KM analysis) } \\
\text { (\$) }\end{array}$ & $\begin{array}{c}\mathbf{N} \\
\text { (censored) } \\
\left(^{*}\right) \\
\end{array}$ \\
\hline \multirow{6}{*}{ N2 (wt) } & \multirow{2}{*}{ control } & \multirow{2}{*}{19} & \multirow{2}{*}{$18,9(0,1)$} & \multirow{2}{*}{28} & \multirow{2}{*}{ A } & $p<0,0001(B)$ & \multirow{2}{*}{$1024(67)$} \\
\hline & & & & & & $\mathrm{p}<0,0001(\mathrm{C})$ & \\
\hline & \multirow{2}{*}{$f t t-1$} & \multirow{2}{*}{17} & \multirow{2}{*}{$16,4(0,1)$} & \multirow{2}{*}{29} & \multirow{2}{*}{ B } & $p<0,0001(A)$ & \multirow{2}{*}{$1590(28)$} \\
\hline & & & & & & $p<0,0001(C)$ & \\
\hline & \multirow{2}{*}{$f t-2$} & \multirow{2}{*}{16} & \multirow{2}{*}{$15,6(0,1)$} & \multirow{2}{*}{31} & \multirow{2}{*}{ C } & $p<0,0001(A)$ & \multirow{2}{*}{$1501(49)$} \\
\hline & & & & & & $\mathrm{p}<0,0001(\mathrm{~B})$ & \\
\hline \multirow{6}{*}{$\begin{array}{c}\text { daf-16 } \\
\text { (mgDf50) }\end{array}$} & \multirow{2}{*}{ control } & \multirow{2}{*}{14} & \multirow{2}{*}{$13,9(0,1)$} & \multirow{2}{*}{24} & \multirow{2}{*}{ D } & $p=0,089(E)$ & \multirow{2}{*}{$806(24)$} \\
\hline & & & & & & $\mathrm{p}<0,0001(\mathrm{~F})$ & \\
\hline & \multirow{2}{*}{$f t t-1$} & 14 & $140(03)$ & 29 & $F$ & $p=0,089(D)$ & $261(0)$ \\
\hline & & & & & & $\mathrm{p}<0,0001(\mathrm{~F})$ & \\
\hline & $f t ?$ & 12 & $128(01)$ & 20 & $\mathrm{E}$ & $p<0,0001(D)$ & 178 (36) \\
\hline & $\pi t-2$ & (10 & $12,0(0,1)$ & 20 & 1 & $p<0,0001(E)$ & $410(00)$ \\
\hline & control & 34 & $313(07)$ & 12 & C & $\mathrm{p}<0,0001(\mathrm{H})$ & 170 \\
\hline & क्षाता & 04 & (ז, ט(ט, & 42 & $\vec{G}$ & $\mathrm{p}<0,0001(\mathrm{~J})$ & $170(117)$ \\
\hline daf-2 & $f t t-1$ & 26 & $254(03)$ & 38 & $\mathrm{H}$ & $p<0,0001(G)$ & $380(18)$ \\
\hline (e1365) & $m-1$ & 20 & $2 v, 4(0, v)$ & 30 & TI & $p=0,623(J)$ & $800(10)$ \\
\hline & $f+2$ & 23 & $234(04)$ & 38 & 1 & $p<0,0001(G)$ & 432 (2) \\
\hline & $m-L$ & 20 & $20,4(v, 4)$ & 30 & 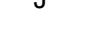 & $p=0,623(H)$ & $402(2<)$ \\
\hline & control & 30 & $286(04)$ & 44 & $K$ & $p<0,0001(L)$ & 2770 \\
\hline & क्षाता & ( & $20,0(0,4)$ & 44 & $x$ & $p<0,0001(M)$ & $211(0)$ \\
\hline$d a f-2$ & $f t t-1$ & 20 & $19.6(0.2)$ & 39 & 1 & $\mathrm{p}<0,0001(\mathrm{~K})$ & $953(0)$ \\
\hline (e1370) & & & & & & $p<0,0001(M)$ & (U) \\
\hline & $f+2$ & 10 & $185(01)$ & ? & & $\mathrm{p}<0,0001(\mathrm{~K})$ & 1) \\
\hline & $m t-2$ & 19 & $18,5(0,1)$ & 39 & IVI & $p<0,0001(L)$ & $1272(4)$ \\
\hline & control & 21 & $215(05)$ & 42 & $N$ & $p<0,0001(0)$ & $10(58)$ \\
\hline & Contur & 21 & $21,0(0,0)$ & 42 & N & $p=0,796(P)$ & (טס) \\
\hline$p d k-1$ & $f t-1$ & 21 & $217(01)$ & 15 & 0 & $\mathrm{p}<0,0001(\mathrm{~N})$ & $186(71)$ \\
\hline (sa709) & $m-1$ & 24 & $24, I(0,4)$ & 40 & 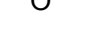 & $\mathrm{p}<0,0001(\mathrm{P})$ & $100(14)$ \\
\hline & $f t t-2$ & 21 & $215(04)$ & 43 & $P$ & $p=0,796(N)$ & $184(72)$ \\
\hline & $m-2$ & 21 & $21, v(0,4)$ & 40 & $r$ & $p<0,0001(O)$ & $104(12)$ \\
\hline & control & 20 & $191(02)$ & 26 & ○ & $p=0,039(R)$ & $(126)$ \\
\hline & प्राtuा & & $19,1(0,2)$ & 20 & 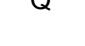 & $p<0,0001(S)$ & $546(136)$ \\
\hline$p d k-1$ & $f t t-1$ & 20 & $195(0.2)$ & 28 & $R$ & $p=0,039(Q)$ & $783(75)$ \\
\hline (mg142) & & & (U), & & & $\mathrm{p}<0,0001(\mathrm{~S})$ & \\
\hline & & & & & & $p<0,0001(Q)$ & \\
\hline & $\mathrm{ftt}-2$ & 11 & $(0,2)$ & 31 & $S$ & $p<0,0001(R)$ & 15) \\
\hline
\end{tabular}




\begin{tabular}{|c|c|c|c|c|c|c|}
\hline Strain & $\begin{array}{l}\text { RNAi during } \\
\text { development }\end{array}$ & $\begin{array}{c}\text { Mean life span } \\
\text { days (+ /-SD) } \\
\text { (@) }\end{array}$ & $\begin{array}{l}\text { Max life } \\
\text { span }\end{array}$ & code & $\begin{array}{c}\text { Statistics } \\
\text { (KM analysis) } \\
\text { (\$) }\end{array}$ & $\begin{array}{c}\mathbf{N} \\
\text { (censored) } \\
\left(^{*}\right)\end{array}$ \\
\hline \multirow{3}{*}{ N2 (wt) } & control & $19,0(0,2)$ & 28 & A & $\begin{array}{l}p<0,0001(B) \\
p<0,0001(C)\end{array}$ & $561(11)$ \\
\hline & $f t-1$ & $16,4(0,3)$ & 25 & B & $\begin{array}{l}p<0,0001(\mathrm{~A}) \\
\mathrm{p}=0,007(\mathrm{C})\end{array}$ & $241(50)$ \\
\hline & $\mathrm{ftt}-2$ & $14,2(0,3)$ & 25 & C & $\begin{array}{l}p<0,0001(A) \\
p=0,007(B)\end{array}$ & 384 (72) \\
\hline \multirow{3}{*}{$\begin{array}{c}\text { daf-16 } \\
\text { (mgDf50) }\end{array}$} & control & $13,0(0,1)$ & 19 & D & $\begin{array}{l}p<0,0001(E) \\
p<0,0001(F)\end{array}$ & 359 (17) \\
\hline & $f t-1$ & $8,7(0,2)$ & 16 & E & $\begin{array}{c}p<0,0001(\mathrm{D}) \\
p=0,153(\mathrm{~F})\end{array}$ & 208 (20) \\
\hline & $f t t-2$ & $7,3(0,3)$ & 18 & $F$ & $\begin{array}{c}p<0,0001(D) \\
p=0,153(E)\end{array}$ & $318(9)$ \\
\hline \multirow{3}{*}{$\begin{array}{c}d a f-2 \\
(e 1365)\end{array}$} & control & $28,6(0,4)$ & 40 & J & $\begin{array}{l}p<0,0001(K) \\
p<0,0001(L)\end{array}$ & $342(6)$ \\
\hline & $f t-1$ & $12,5(0,2)$ & 35 & K & $\begin{array}{l}p<0,0001(J) \\
p=0,614(L)\end{array}$ & 620 (95) \\
\hline & $f t t-2$ & $11,4(0,6)$ & 33 & L & $\begin{array}{l}p<0,0001(J) \\
p=0,614(K)\end{array}$ & $348(0)$ \\
\hline \multirow{3}{*}{$\begin{array}{c}d a f-2 \\
(e 1370)\end{array}$} & control & $26,1(0,3)$ & 39 & G & $\begin{array}{l}p<0,0001(H) \\
p<0,0001(I)\end{array}$ & 494 (13) \\
\hline & $f t-1$ & $12,0(0,2)$ & 24 & $\mathrm{H}$ & $\begin{array}{l}p<0,0001(G) \\
p<0,0001(I)\end{array}$ & $425(68)$ \\
\hline & $\mathrm{ftt}-2$ & $8,4(0,4)$ & 32 & I & $\begin{array}{l}p<0,0001(G) \\
p<0,0001(H)\end{array}$ & $435(0)$ \\
\hline
\end{tabular}


ftt-1 RNA level

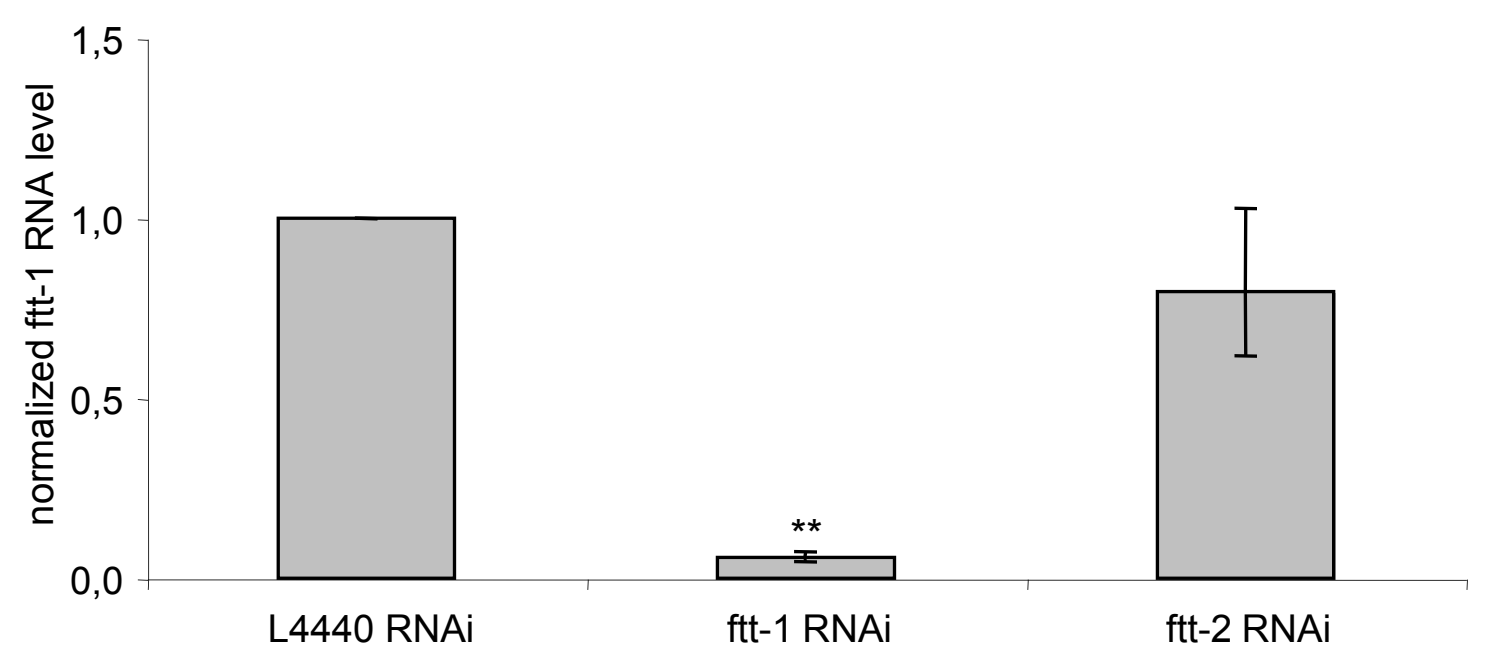

ftt-2 RNA level

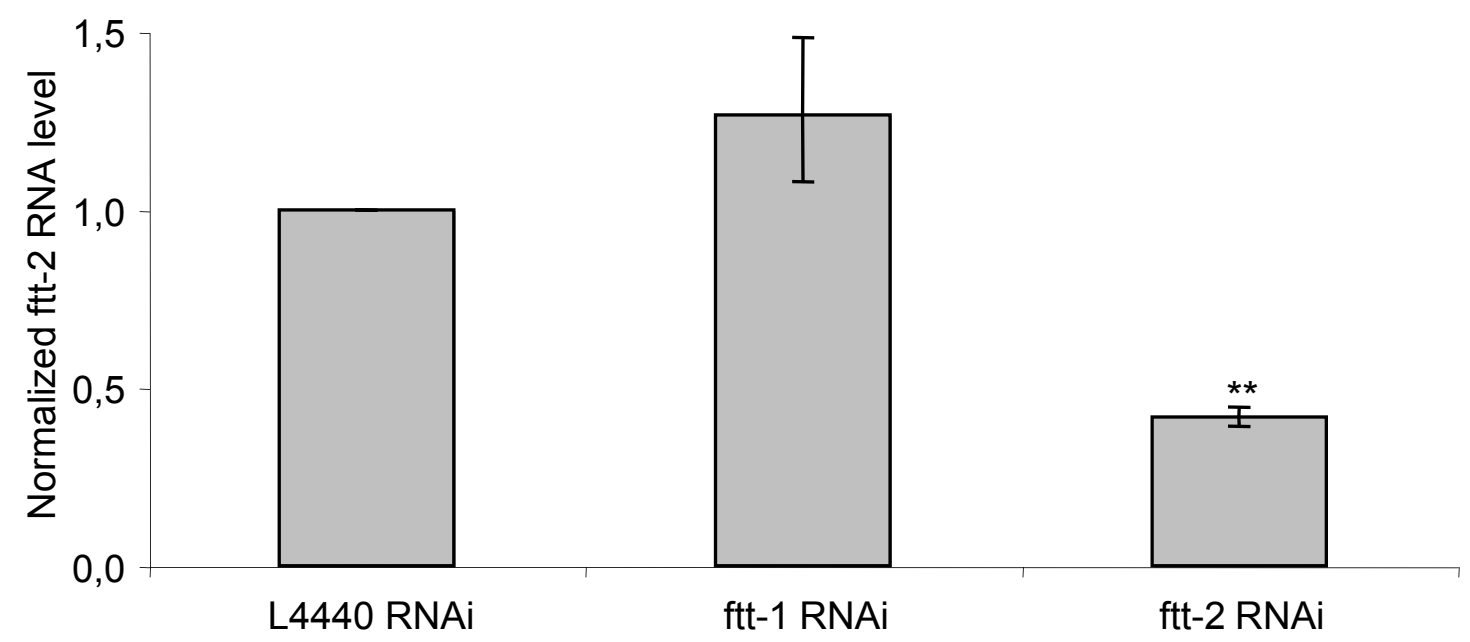


(A)

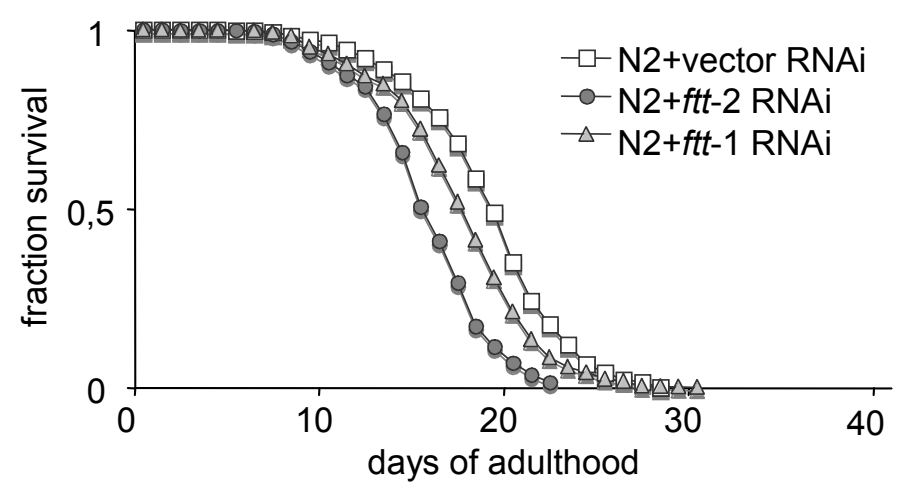

(C)

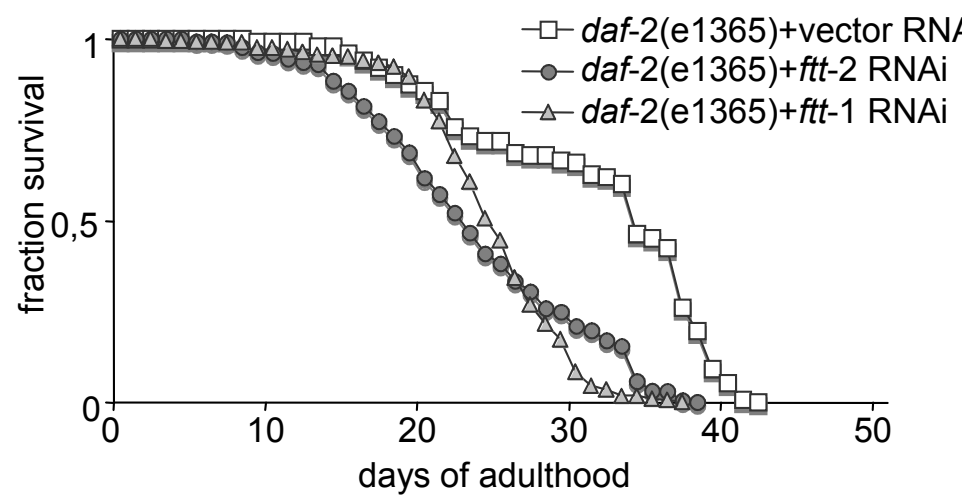

(E)

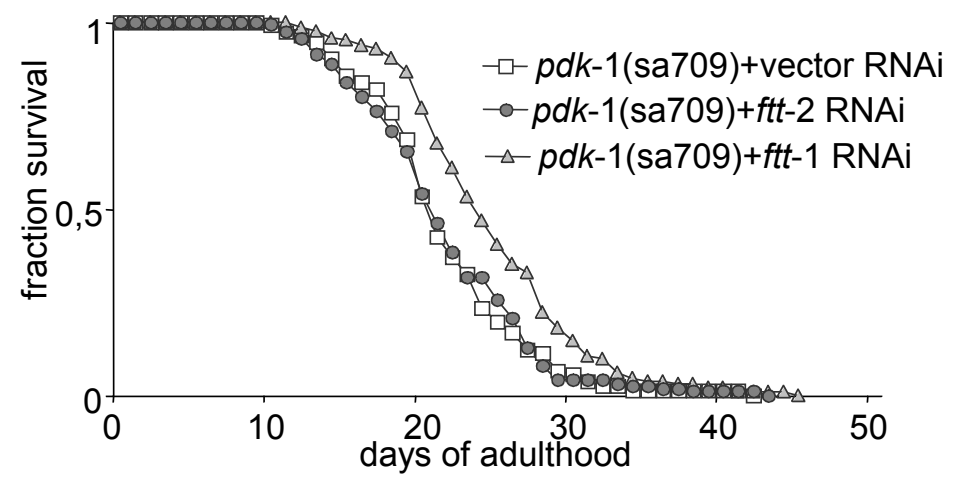

(B)

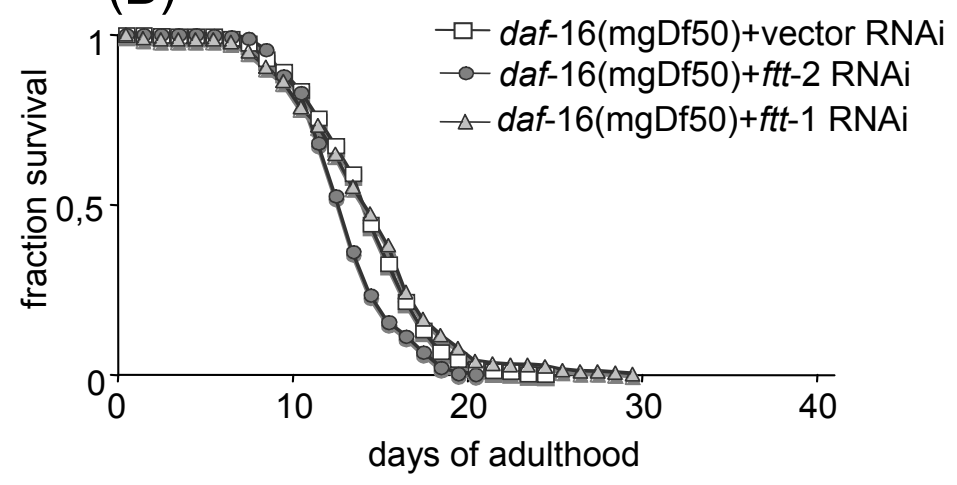

(D)

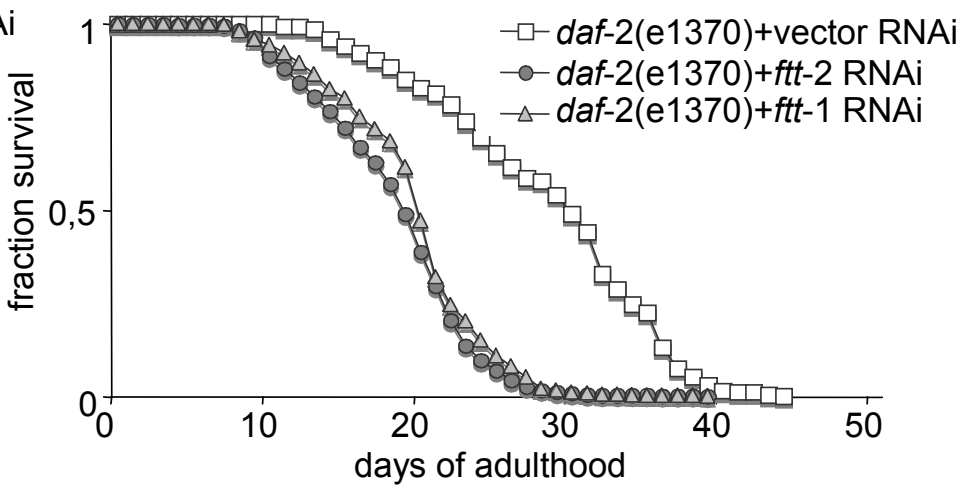

(F)

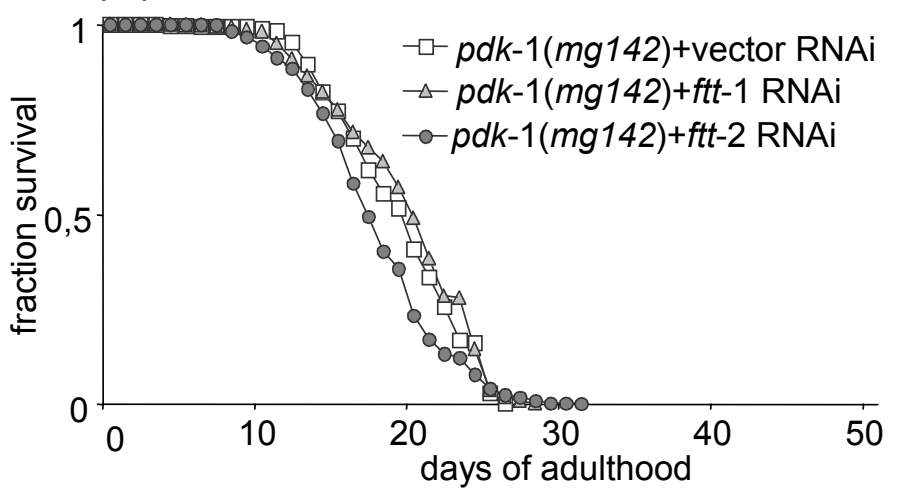



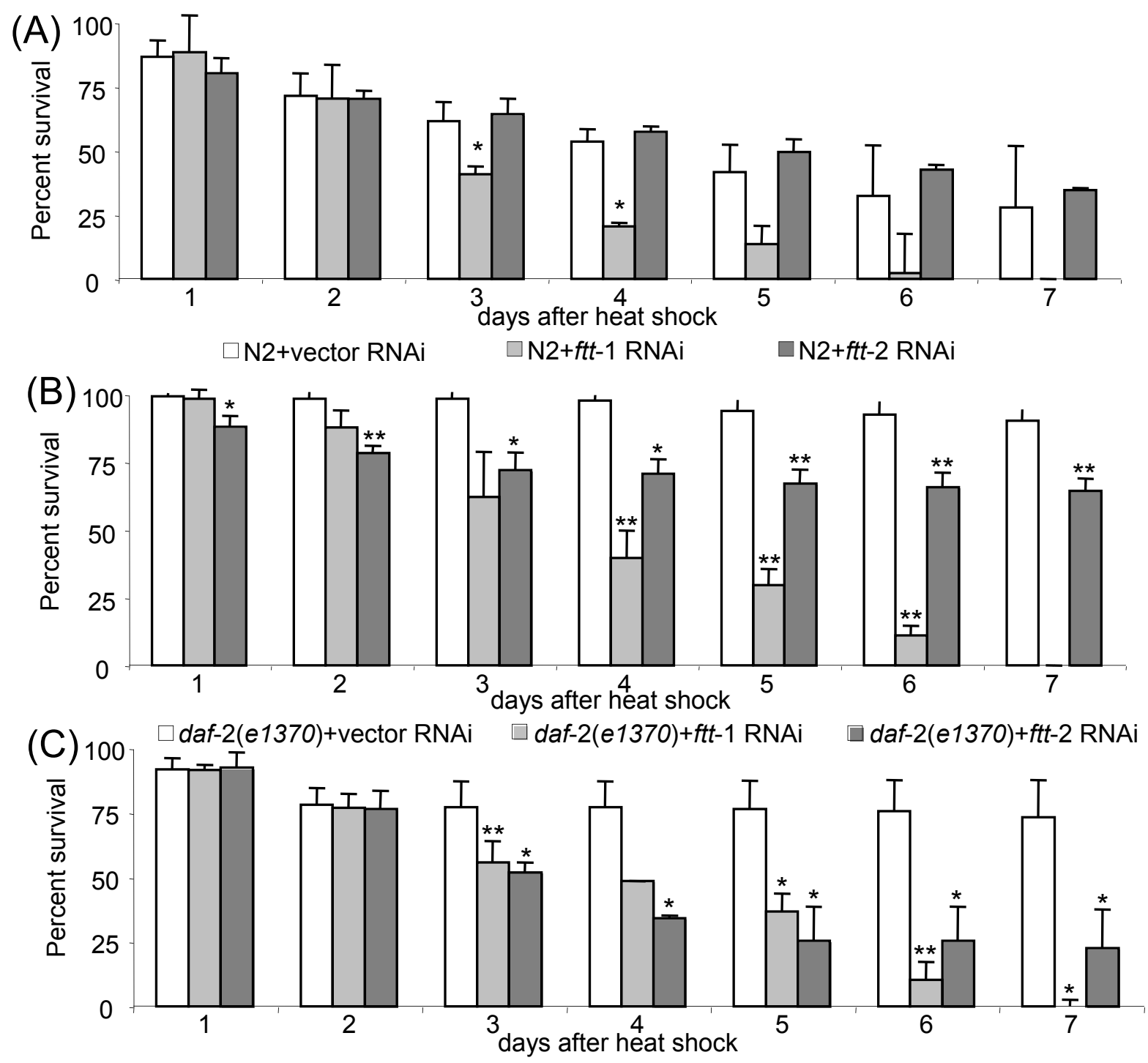

(D) $100 \square$ daf-2(e1365)+vector RNAi $\square$ daf-2(e1365)+ftt-1 RNAi $\square$ daf-2(e1365)+ftt-2 RNAi
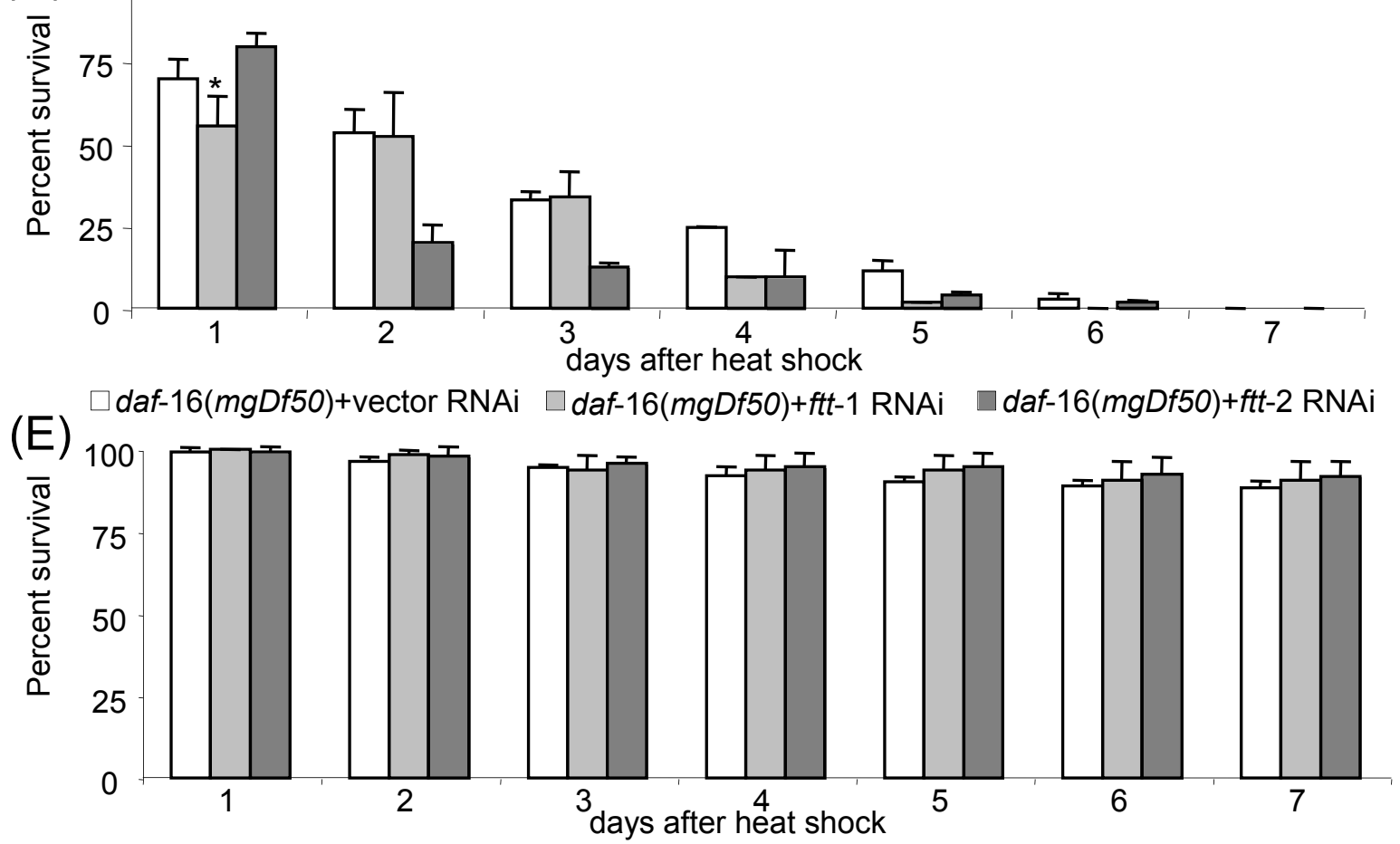

$\square p d k-1(s a 680)+v e c t o r$ RNAi $\quad \square p d k-1(s a 680)+f t t-1$ RNAi $\quad \square p d k-1(s a 680)+f t t-2$ RNAi 


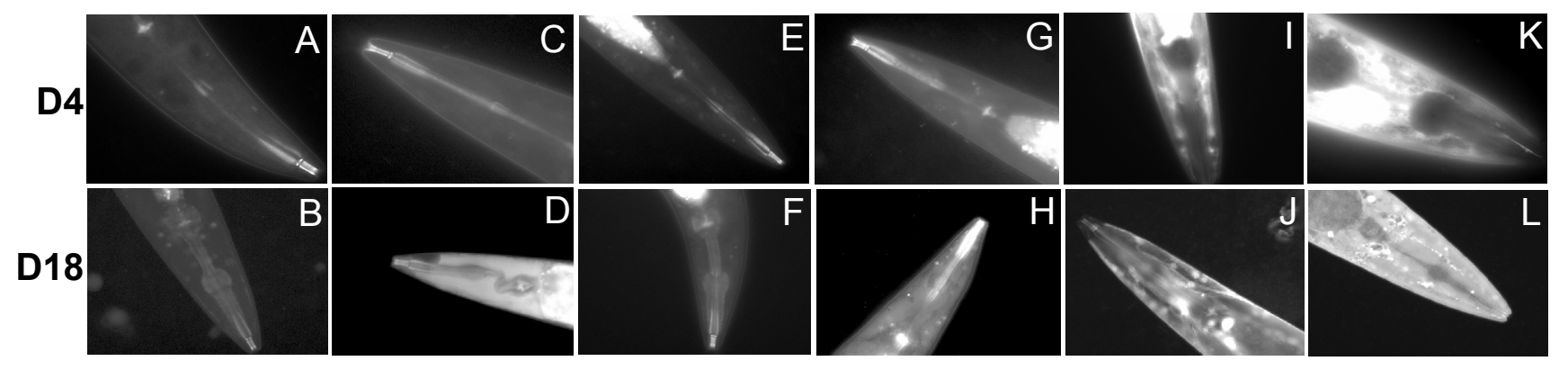


(M)

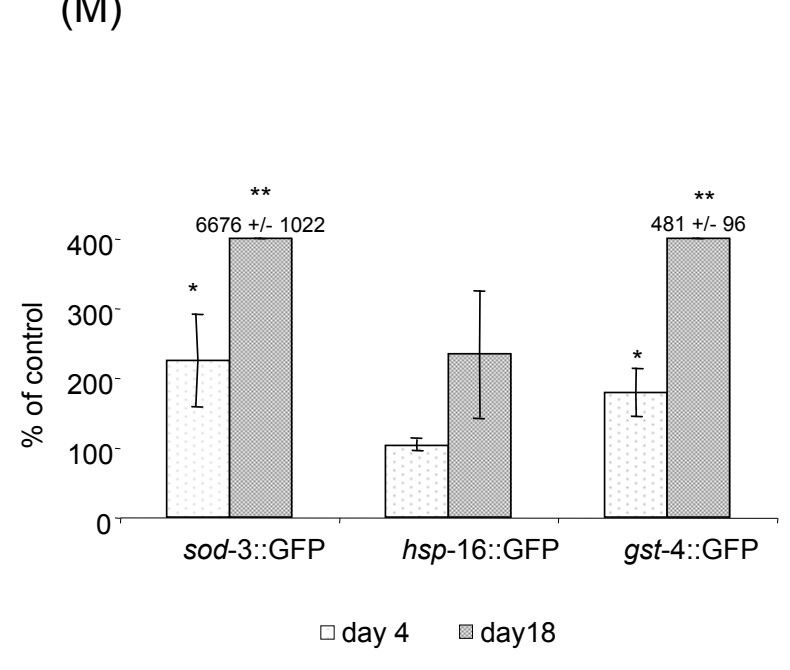

Figure(s)

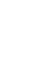




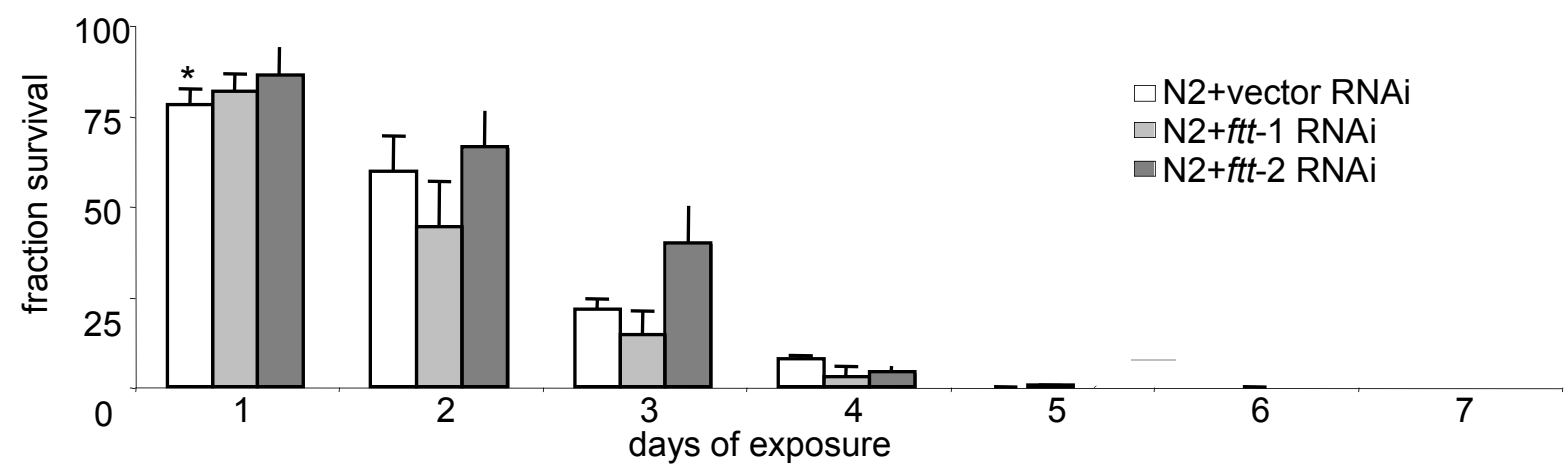

(A)

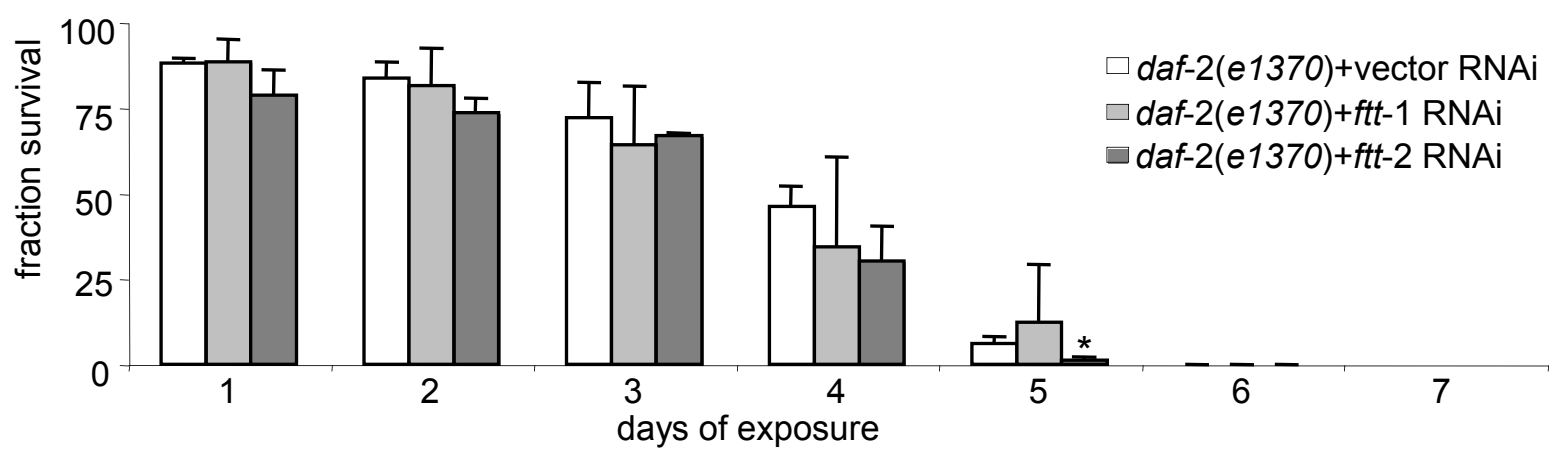

(B)

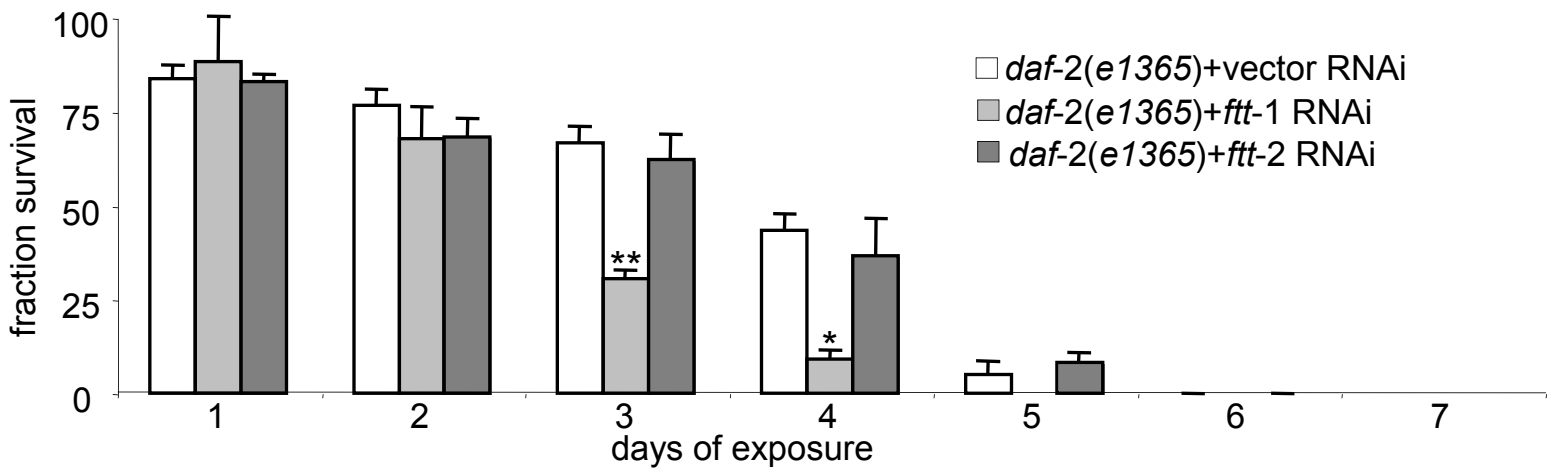

(C)

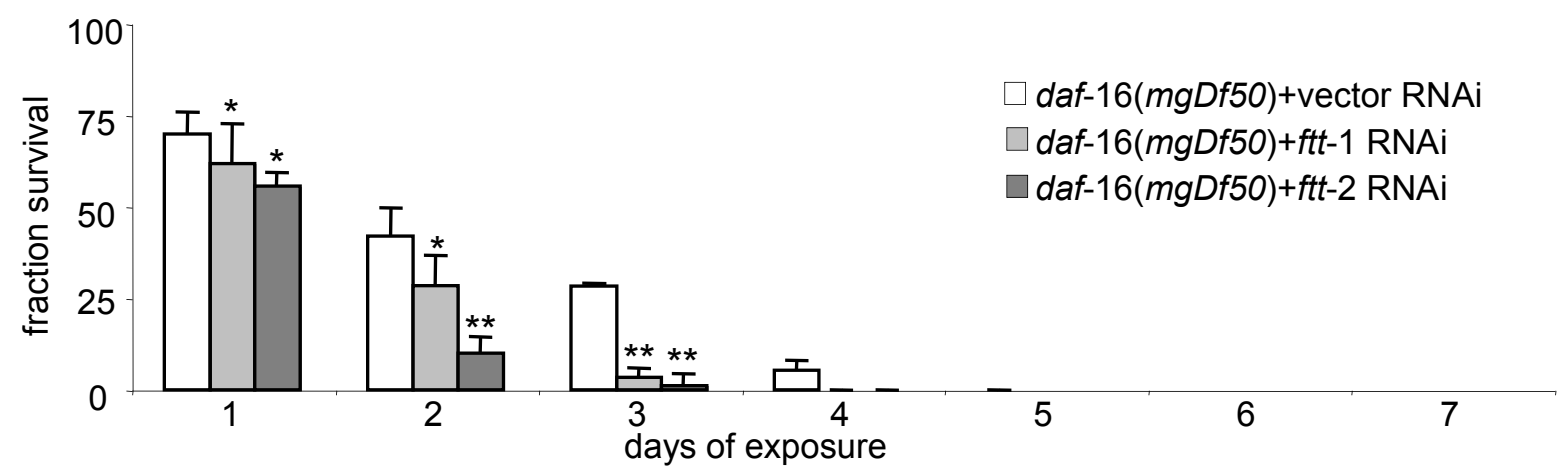

(D) 


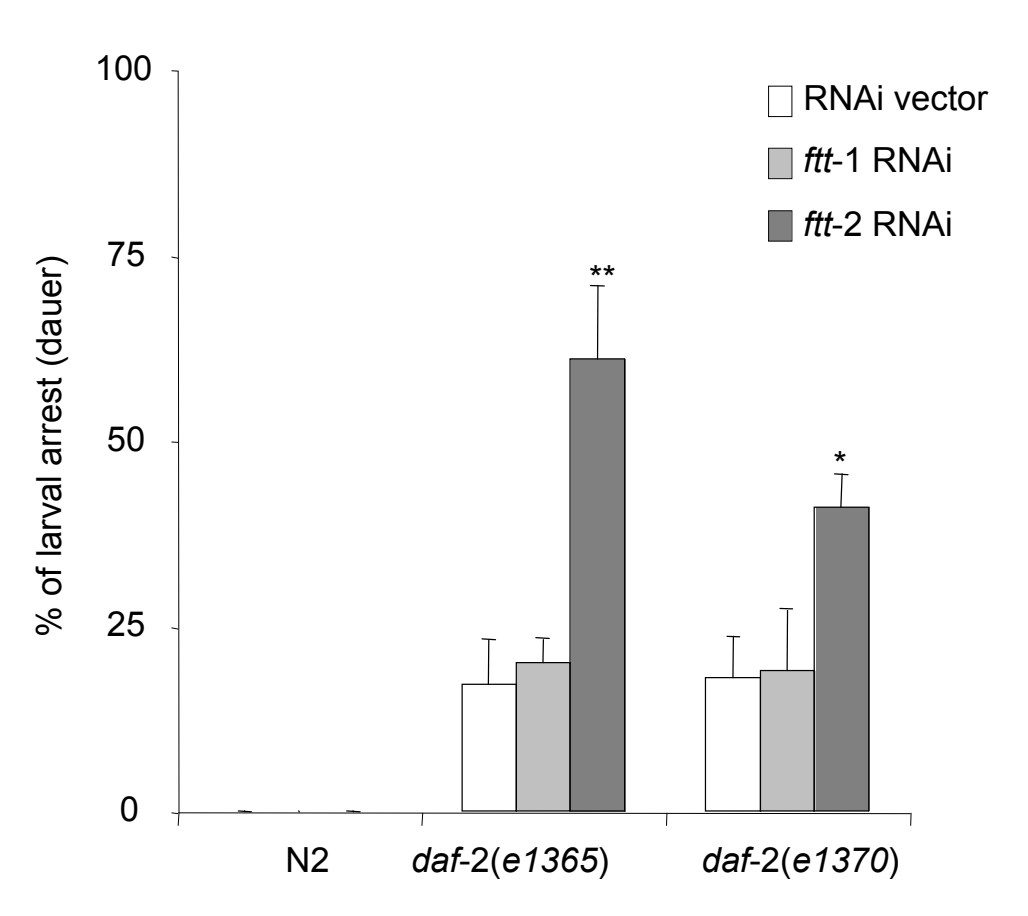

Figure(s) 
(A)

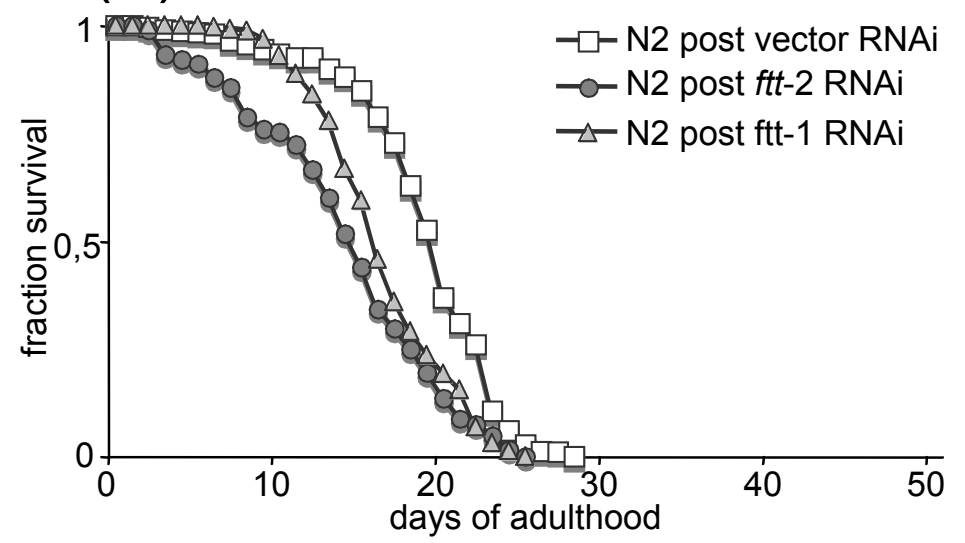

(C)

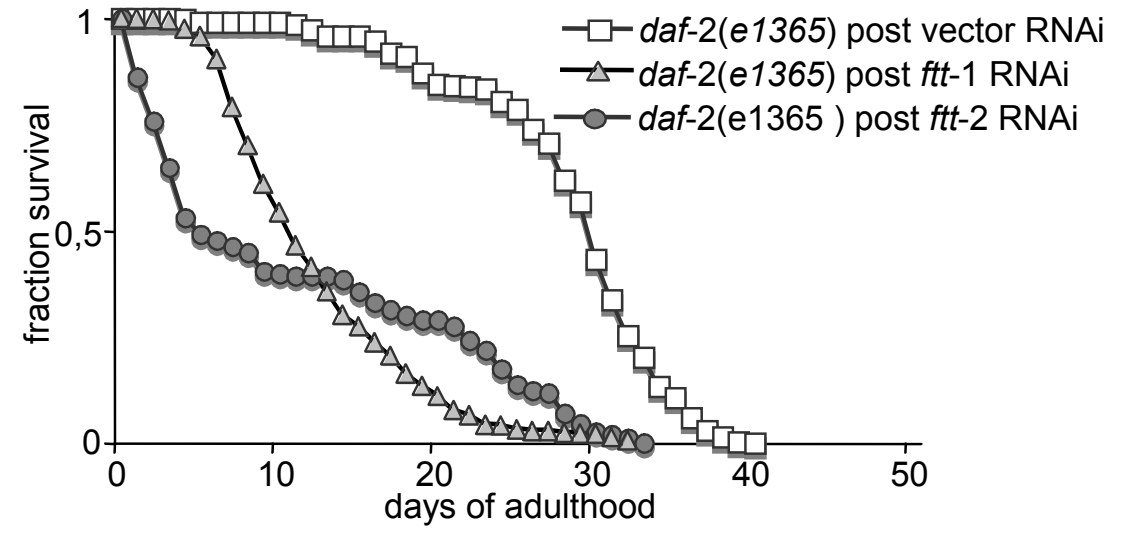

(B)

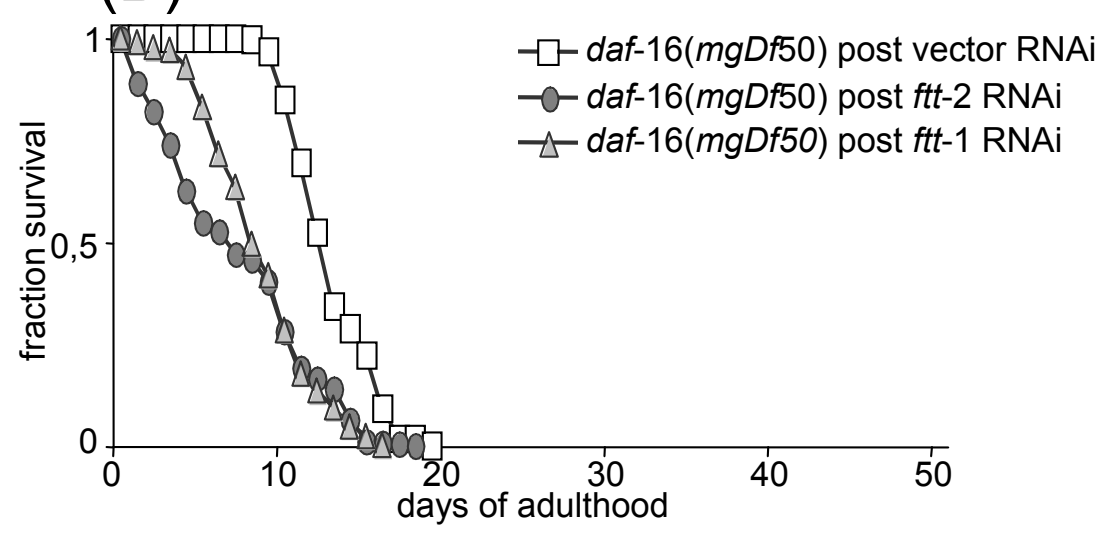

(D)

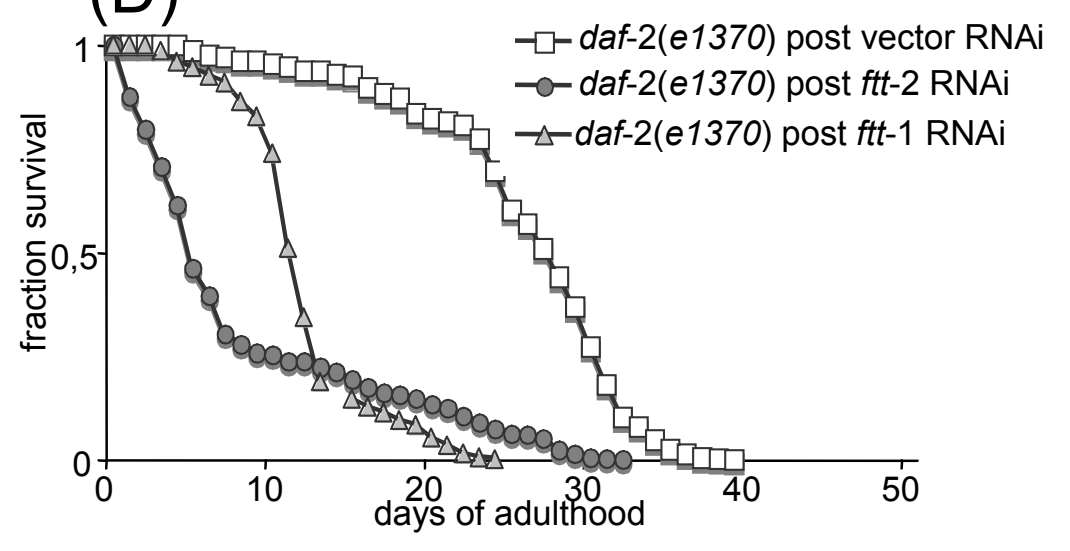




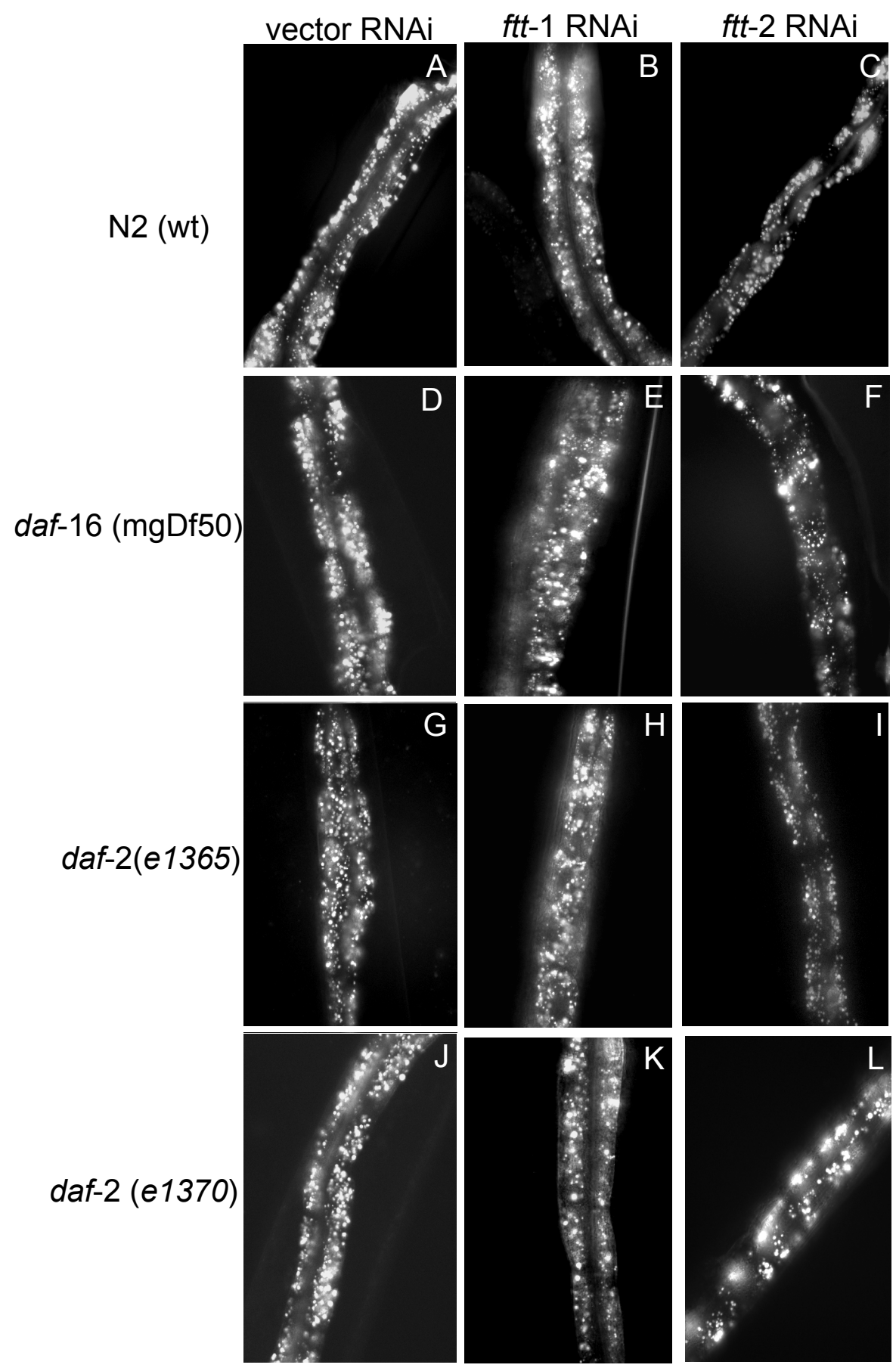


M

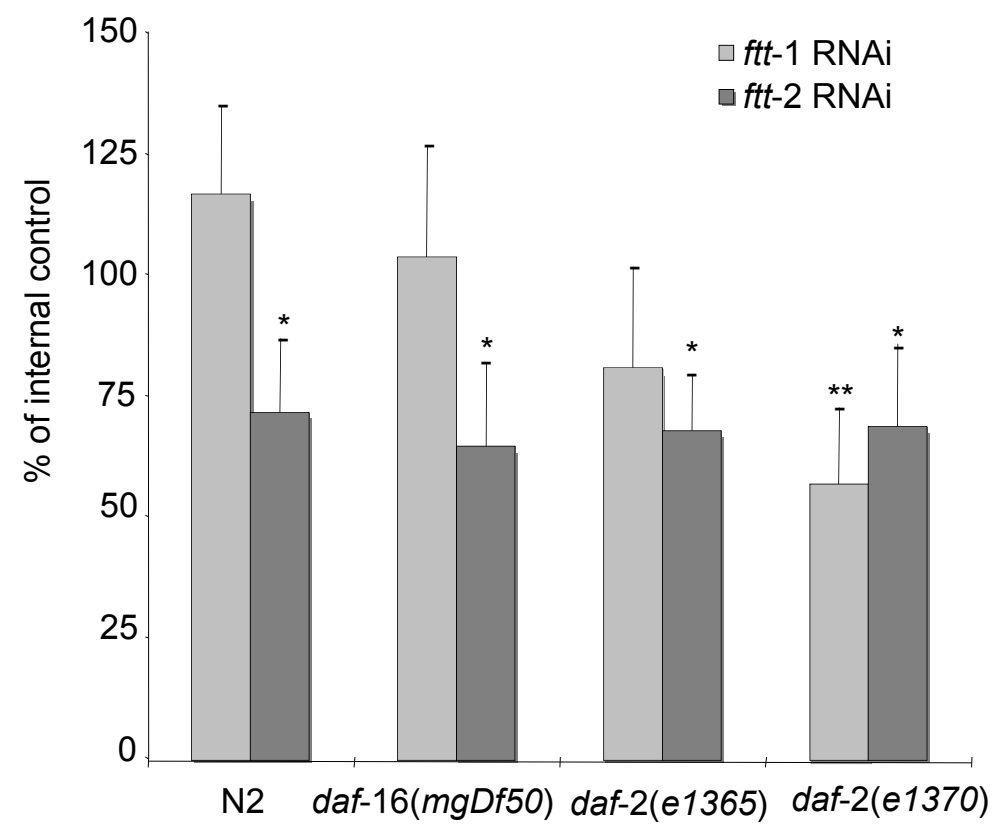




\section{No Stress (A)}

\section{Stress (B)}

IGF-1/DAF-2

1

14-3-3/FTT-1

PDK1
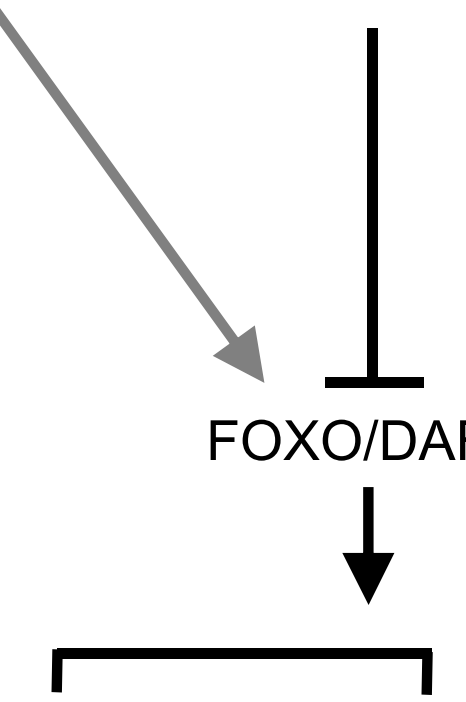

- Development

- Lipid accumulation -Antioxidant and Chaperone genes (SOD-3, HSP-16)

- Life span

- Dauer
IGF-1/DAF-2

FOXO/DAF-16

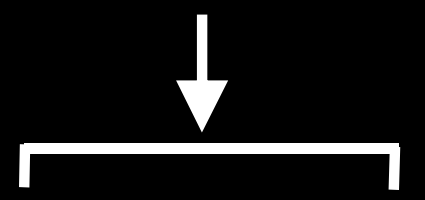

\section{- Thermotolerance \\ - Thermotolerance \\ - Chaperone genes \\ - Longevity}

(a)

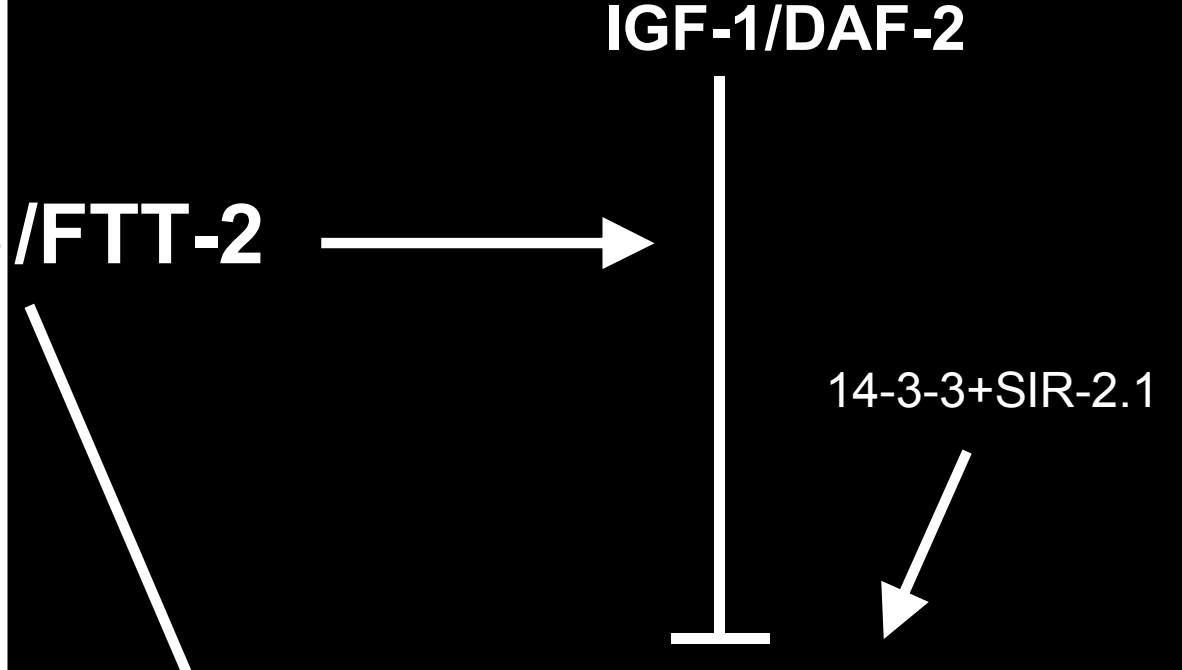

- Oxidative stress

resistance (Menadione) 14-3-3/FTT-2

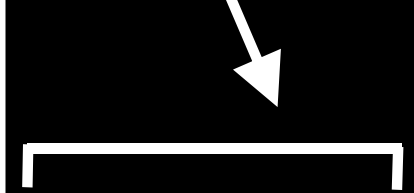
- Lipid accumulation
- Oxidative stress resistance (GST-4) - Life span
- Development

